بررسى مقايسهاى تأثير كرم مهبلى درمنه و كلوتريمازول بر عفونت كانديدايى مهبل بر روى زنان

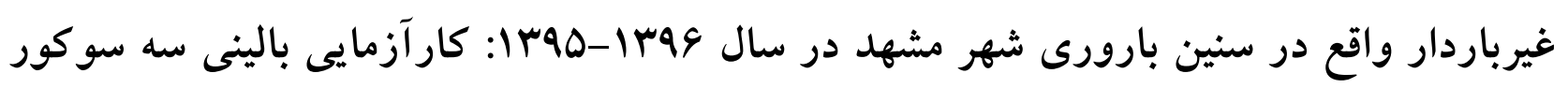

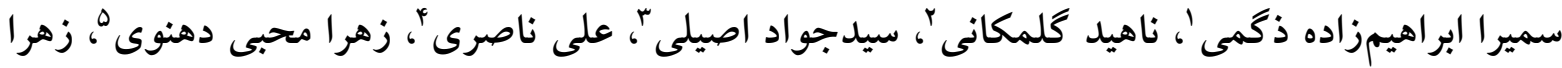

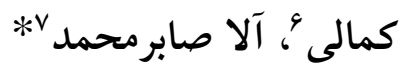

1- عضو هيأت علمى گروه مامايى، دانشجوى دكتراى بهداشت بارورى، دانشكده يرستارى و مامايى، دانشخاه

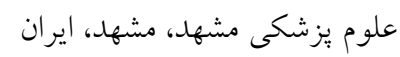

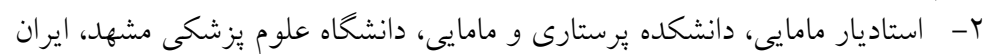

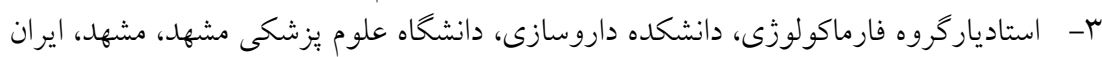

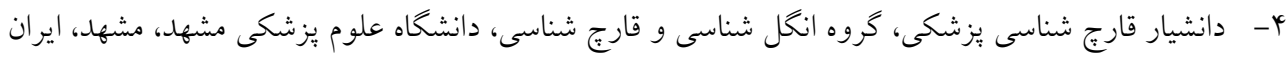

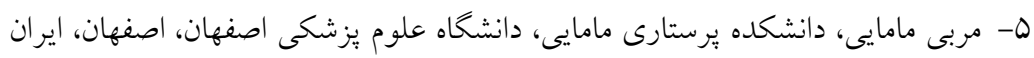

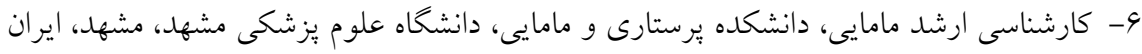

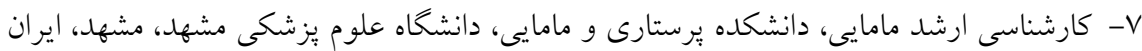

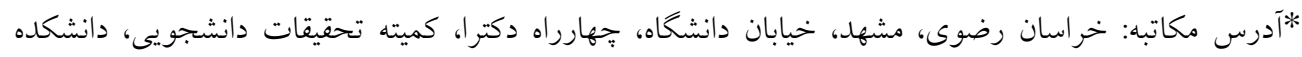

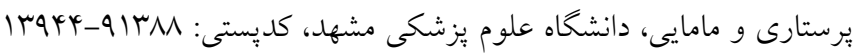

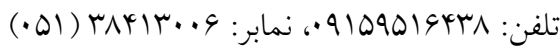
يست الكترونيك:

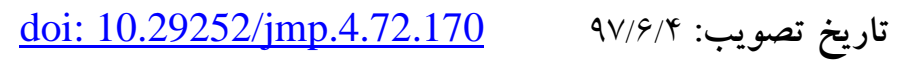

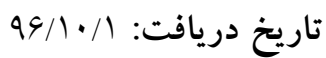

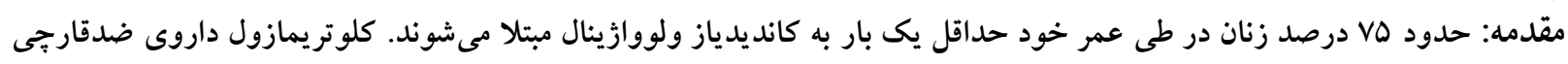

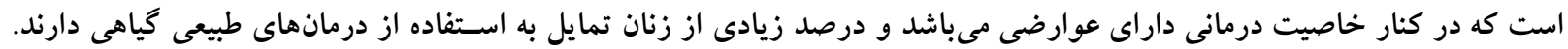

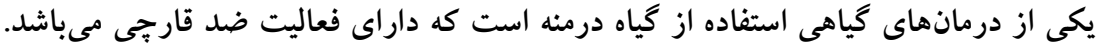

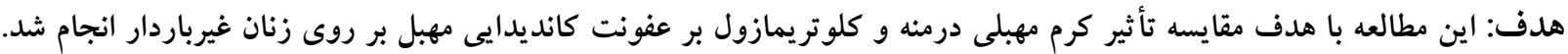

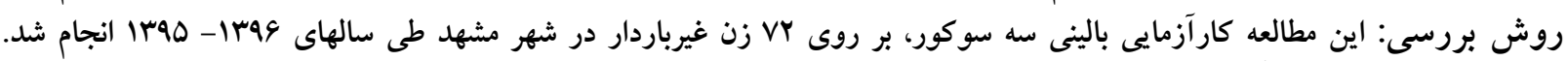

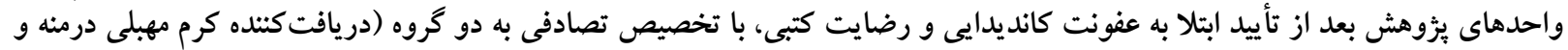

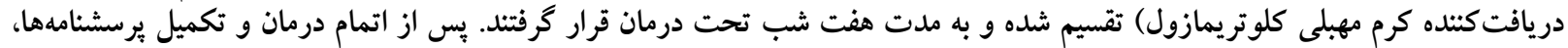

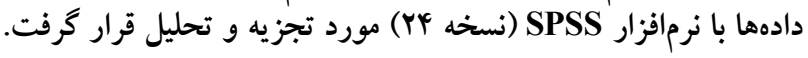

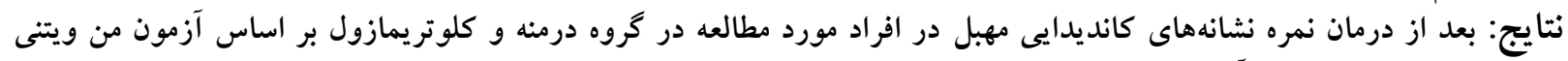

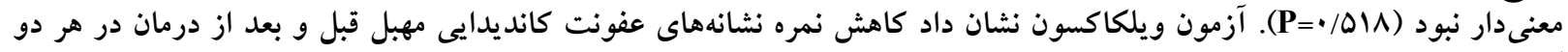

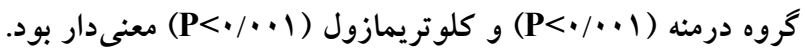

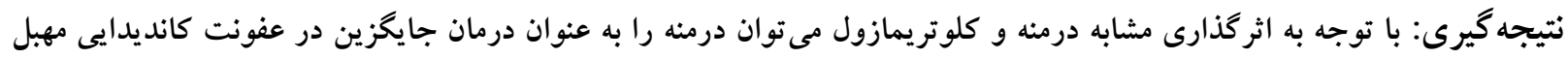

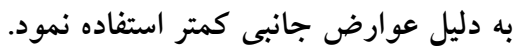
كل 
بررسى مقايسهاى تأثير ...

از علائم ترشح است كه ترشحات مىتواند آبكى تا غليظ و

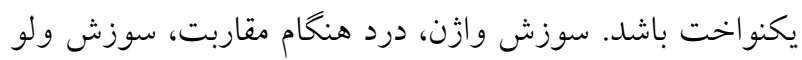

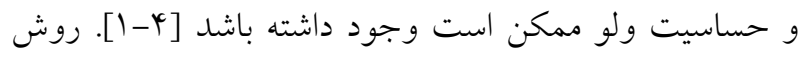

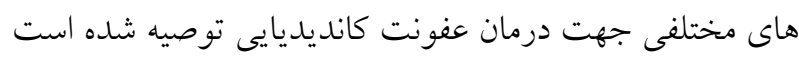
كه از بين روشهاى دارويى، دارئ داروهاى موضعى آزول، متداول

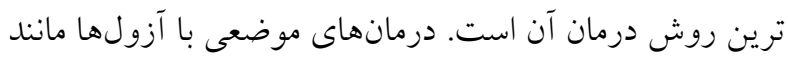

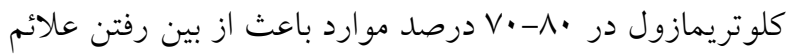

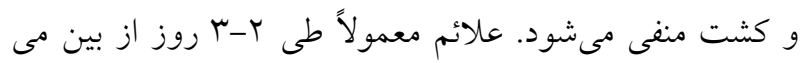

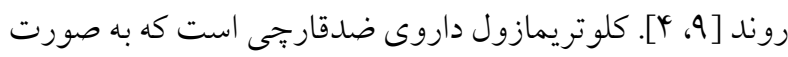

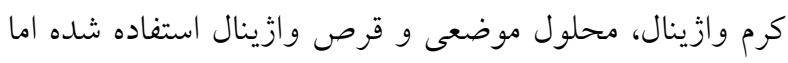
در كنار خاصيت درمانى داراى عوارضى همجيون كهير، تاول،

سوزش، خارش و ساير علائم تحريكى است [•l].

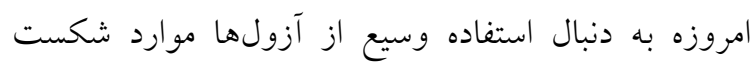
درمان و مقاومت كونههاى مختلف كانديدا نسبت به اين تركيبات دارويى در حال افزايش است كه اين مسأله منجر به منه

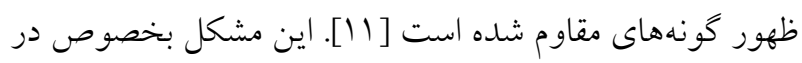

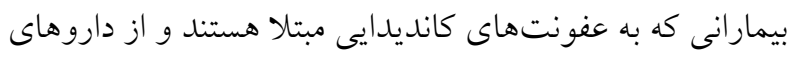
فلوكونازول، كلو تريمازول و كتوكونازول استفاده مى كنند، بسيار

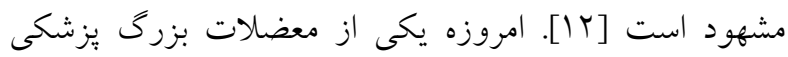

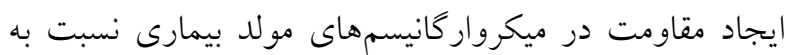

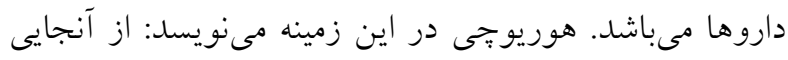

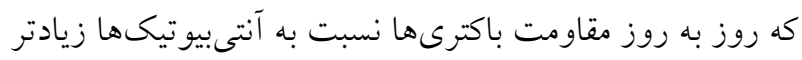
مىشود، نياز فورى به كشف يا توسعه داروهاى جديد و مؤثر براى درمان اين عفونتها وجود دارد [سا].

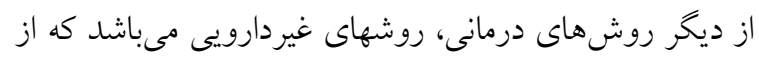

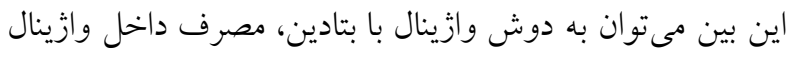

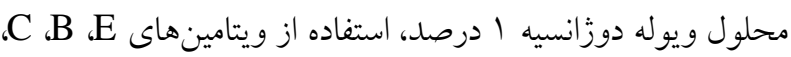

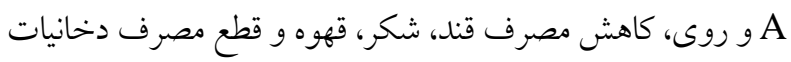

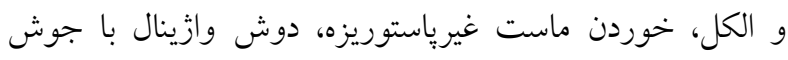

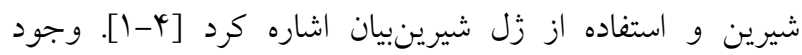
محدوديتهايى نظير تعداد كم انواع داروهاى ضد قارجيى، سمى آنسي

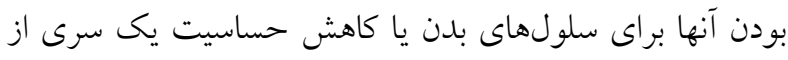

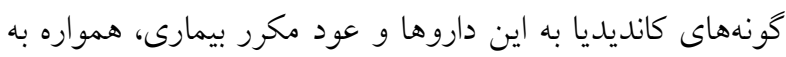
عنوان معضل اساسى در درمان اين بيمارىها مطرح بودهاند [1]".

لاكتوباسيلوس، شايعترين فلور طبيعى وازن است كه در

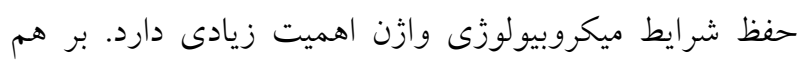

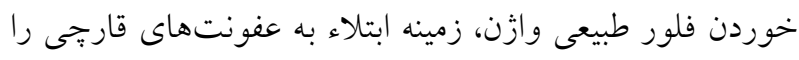

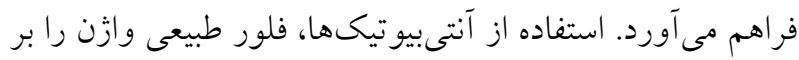

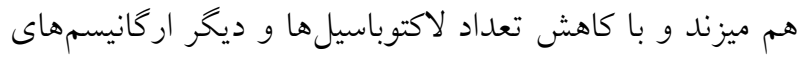

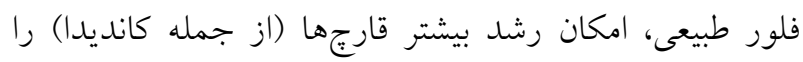

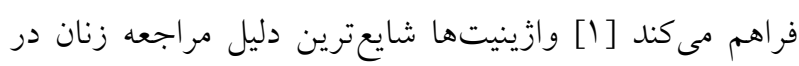

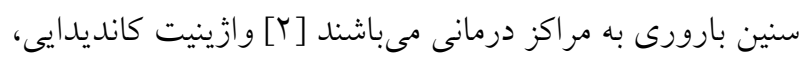
دومين عفونت شايع وازن است [ب، بَّ].

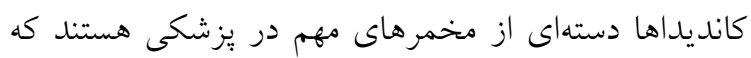

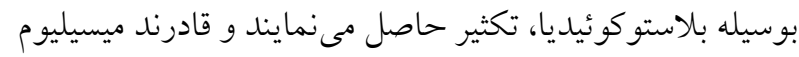

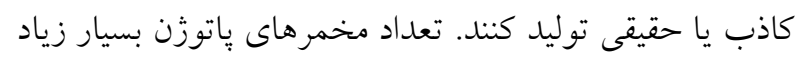

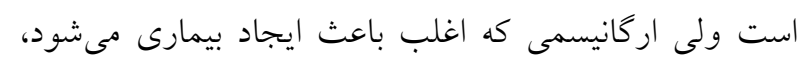

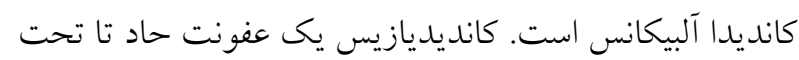

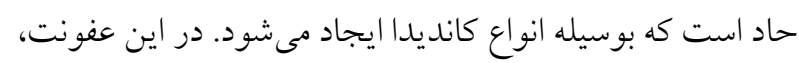

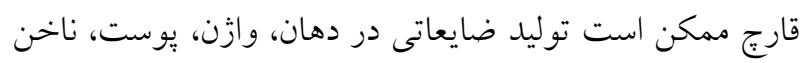

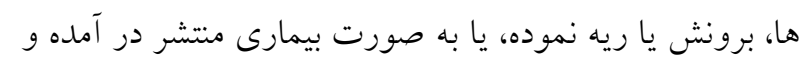

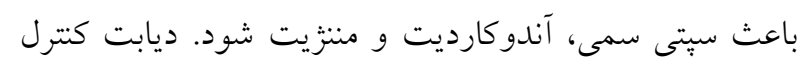

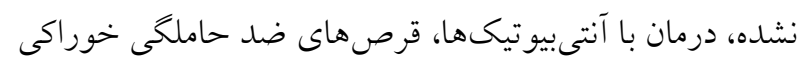

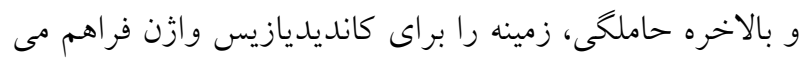

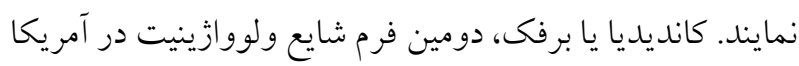
است (

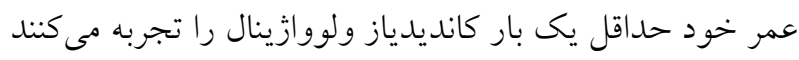

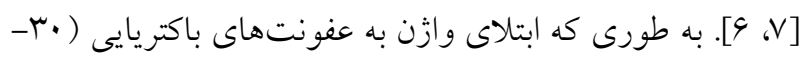

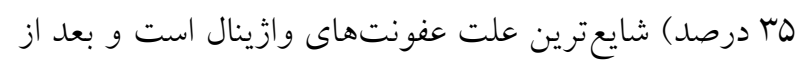

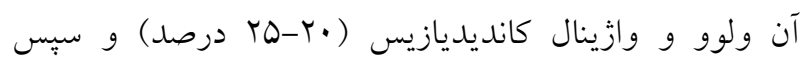

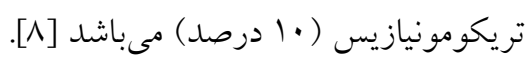

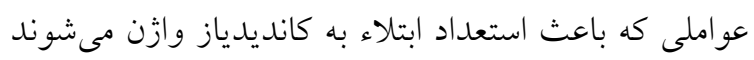

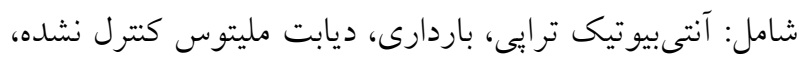

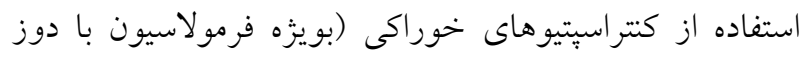

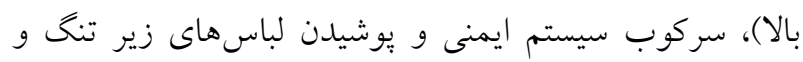

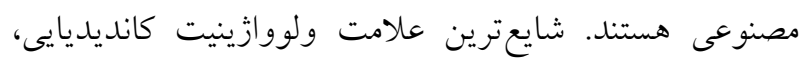

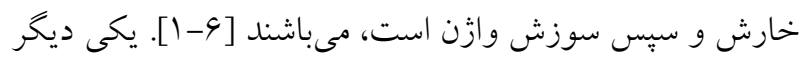


كونههاى كانديدا آلبيكنز مقاوم و حساس به آزولها انجام دادند.

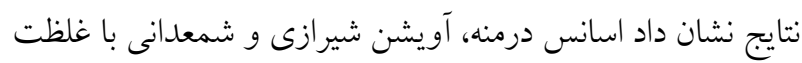
هاى مشابه بر كونههاى كانديدايى حساس و مقاوم به داند دارو مؤثر

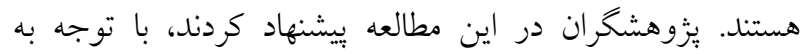

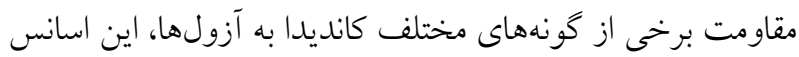

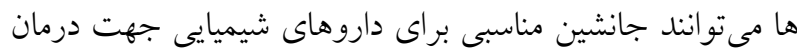

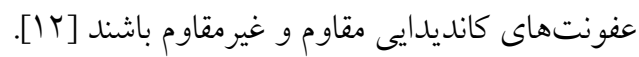

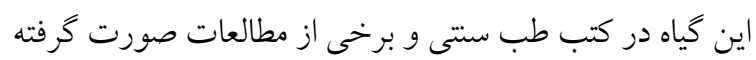

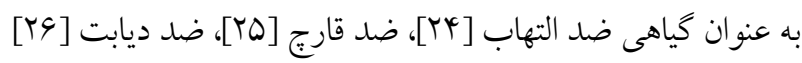

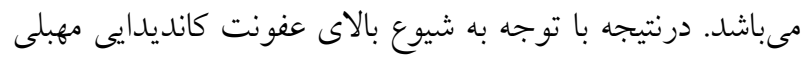

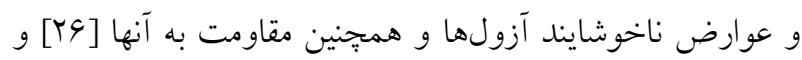

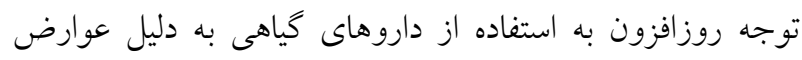

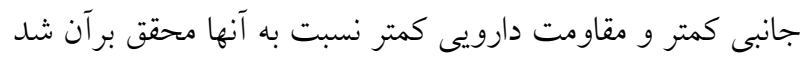

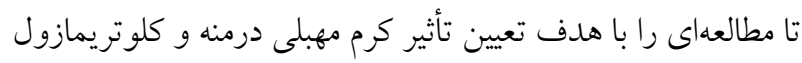
بر عفونت كانديديايى مهبل در زنان غير باردار انجام دهد.

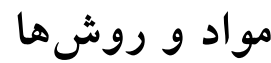

اين

(IRCT2016121831454N1 و سه سوكور بر روى كليه زنان

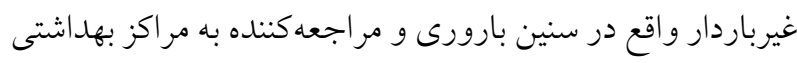

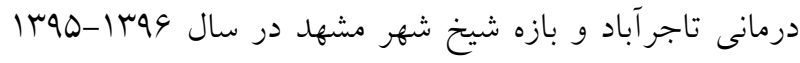

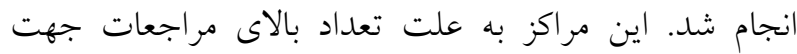

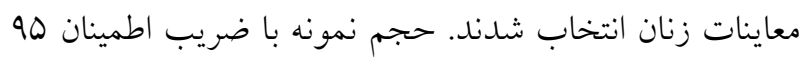

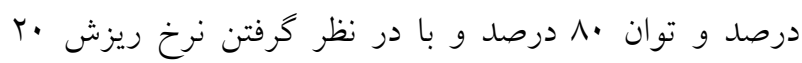

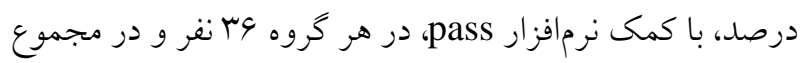

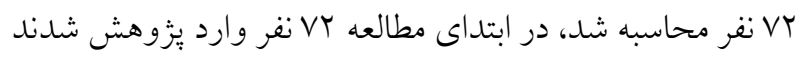

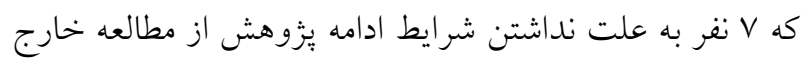

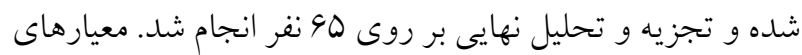

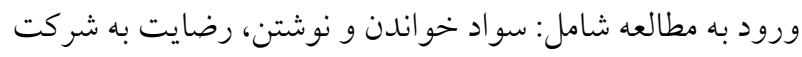

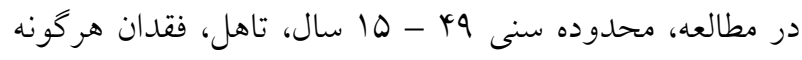

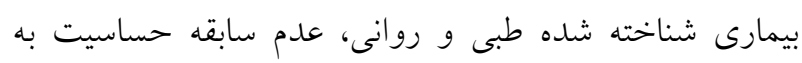

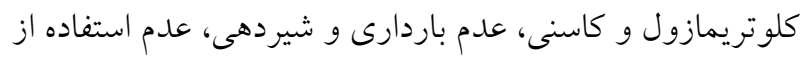
IUD

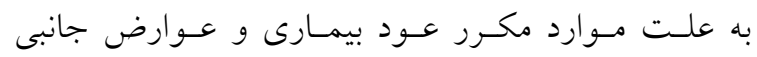

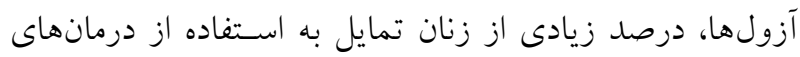

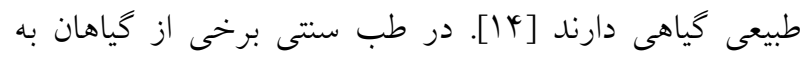

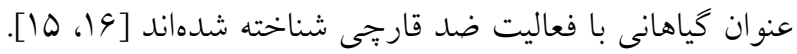

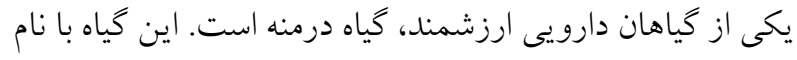
محلى جوشن و نام فارسى درمنه ايرانى و نام علمى آرتميإيرسيكا (Artemisa persica)

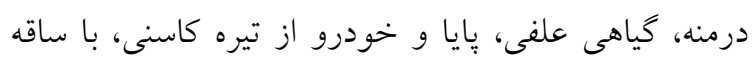

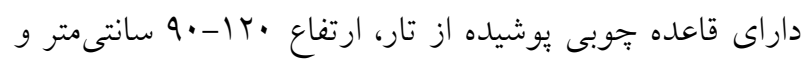

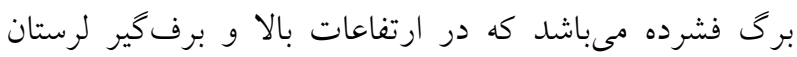

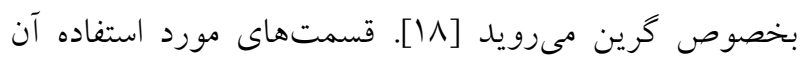

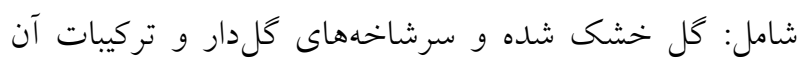

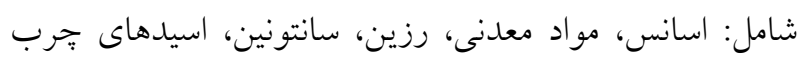

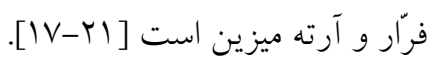

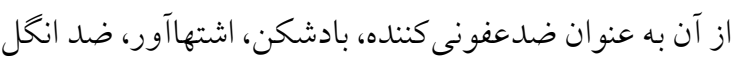

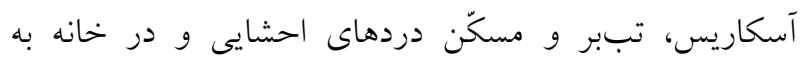

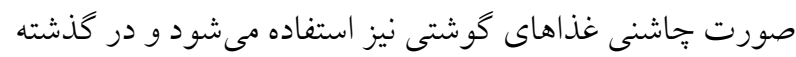
براى تسكين دردهاى عصبى و به عنوان تسهيل كننده انقباضات

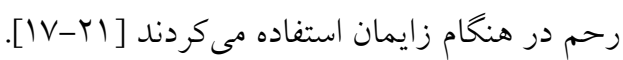

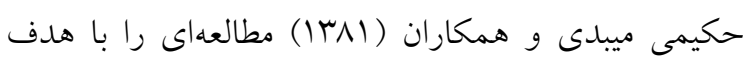
بررسى اثر اسانس درمنه در درمان كانديدا در محيط كشت ميل انجام

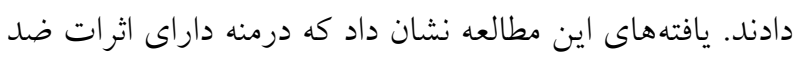

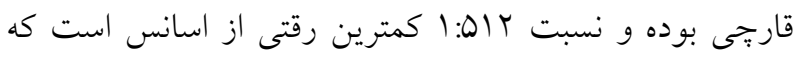

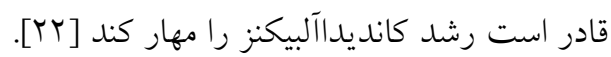

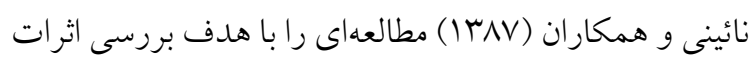

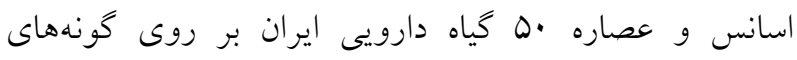

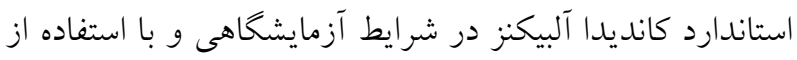

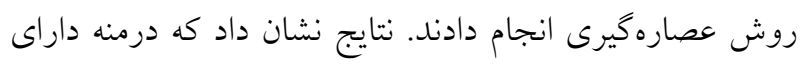

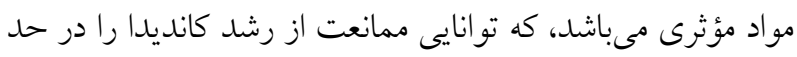

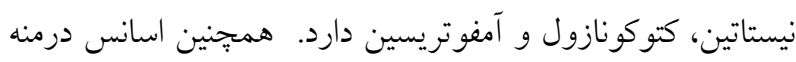

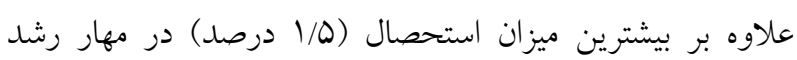

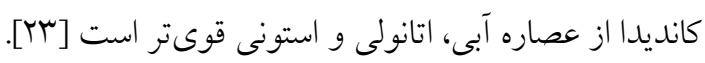

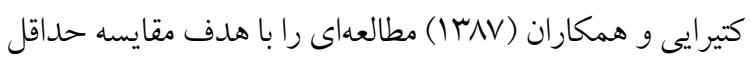

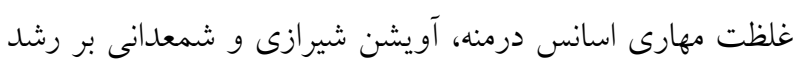


مشخص شدن نتيجه كشت اوليه روى محيط SC، مقدارى از كلنىها به صورت سفرهاى روى محيط افتراقى كروم آثار Himedia

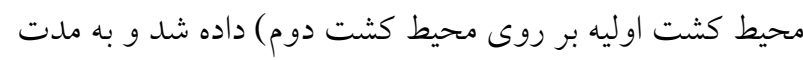

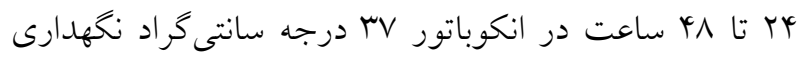

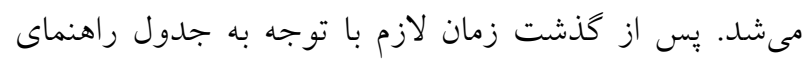

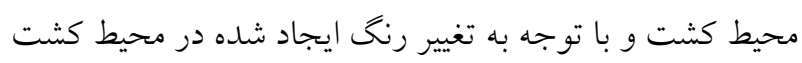

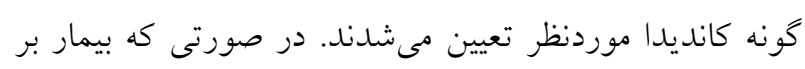

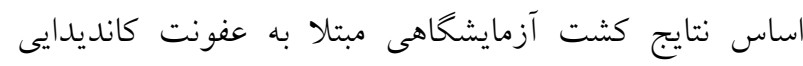

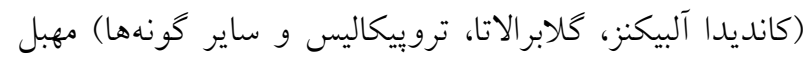

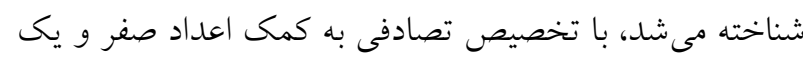

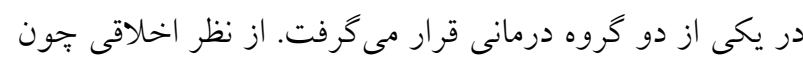

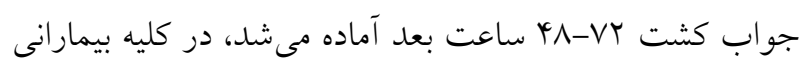

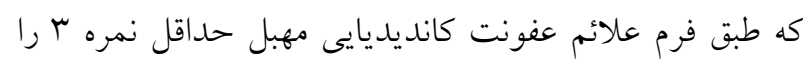

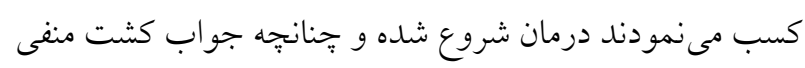

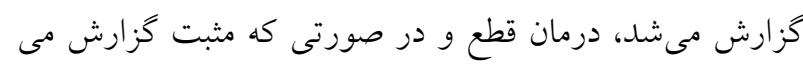

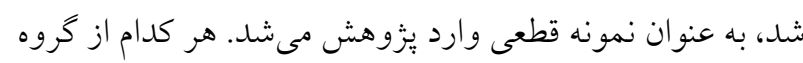

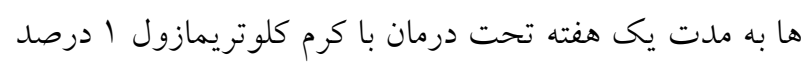

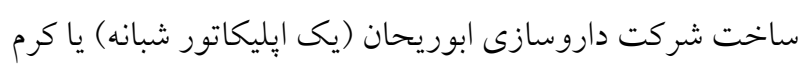

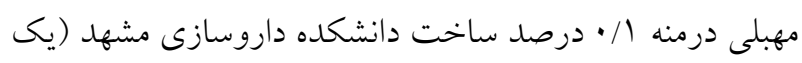

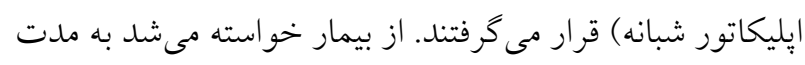

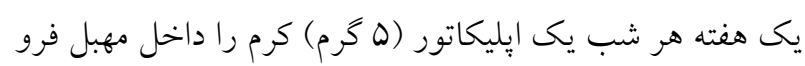

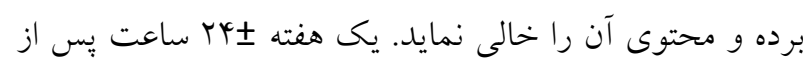

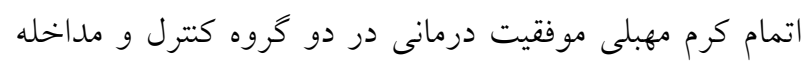

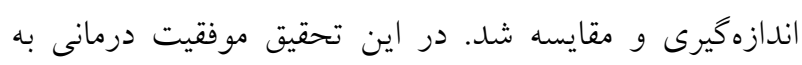

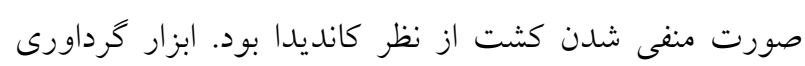

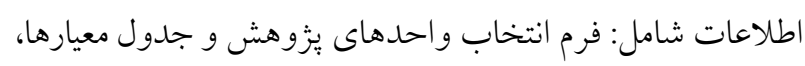

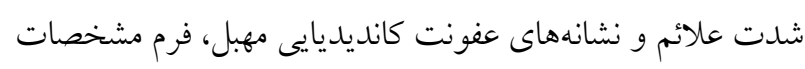

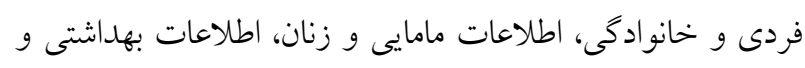

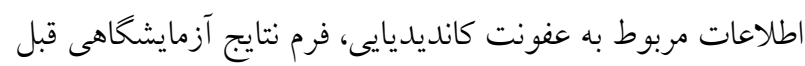

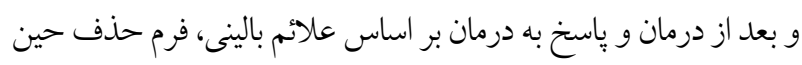
يُزوهش، جّى ليست مصرف دارو و علائم بيمارى در طول دوره درمان

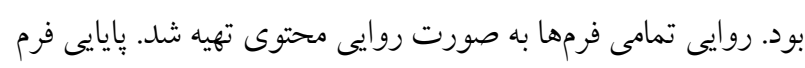

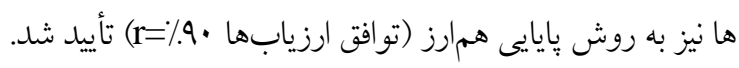

ولووازينيت كانديدايى راجعه، عدم استفاده از ضد قارجهاى

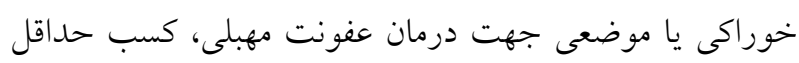

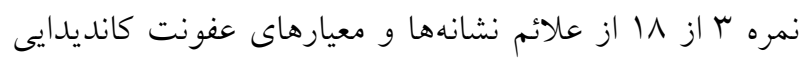

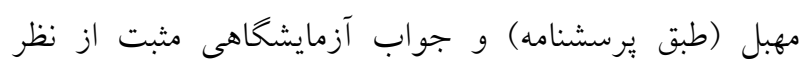

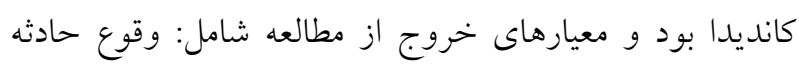

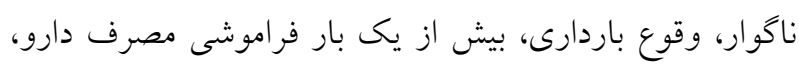

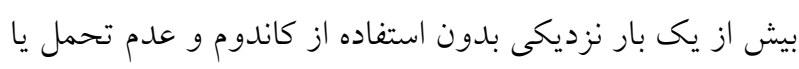

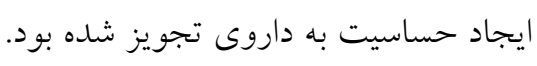
با رعايت نكات اخلاقى و كسب موافقت كتبى از بيمار، ابتدا

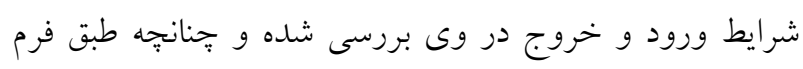

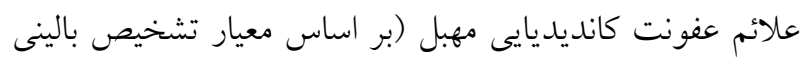

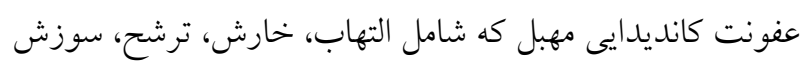

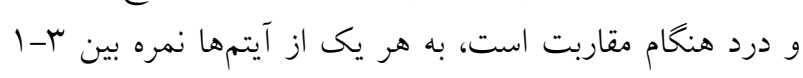

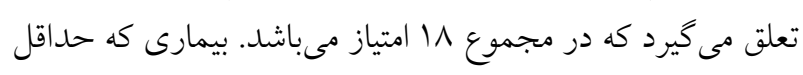

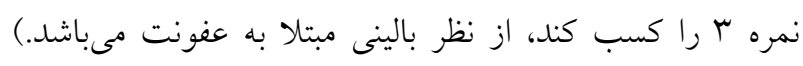

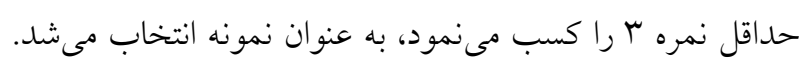

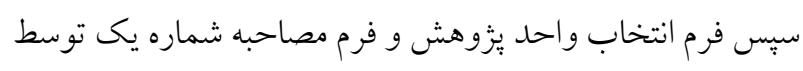

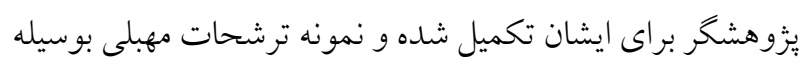

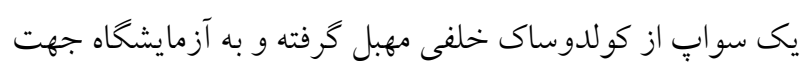
انجام كشت در محيط كشت سابورودكستروز آكار و انجام

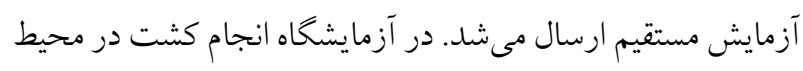

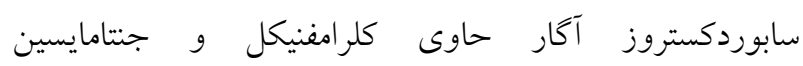
Sabouraud

Dextrose ) (Agar+Cloramphenicol+Gentamicine-2059 شركت conda انجام شد و پِ از رشد كلونىهاى كانديدايى

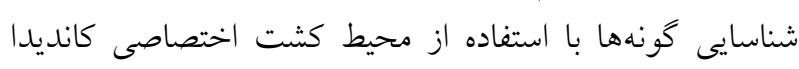
(HiCrome Candida Diffrential Aagar1297A-india) ساخت شركت Himedia انجام شد. بلافاصله بِ از دريافت

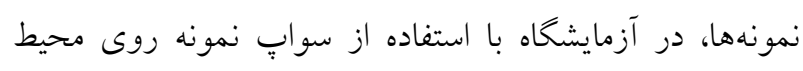

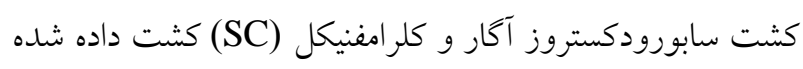

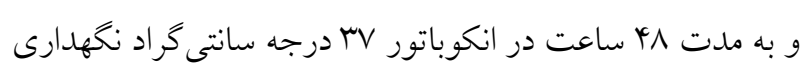

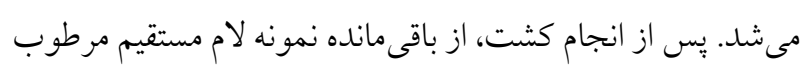

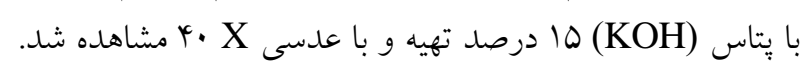

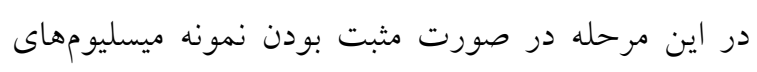

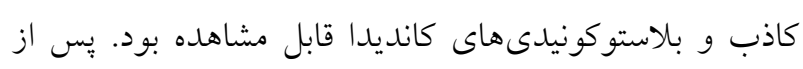


اطلاعات جرمى در محدوده جرمى ها تا هQ 4 كرده است.

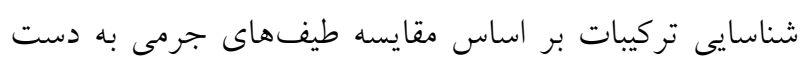
آمده با طيف جرمهاى استاندارد كتابخانهى دستخاه

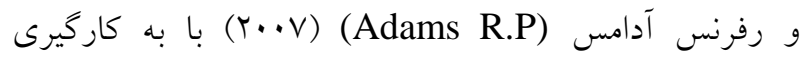
يارامترهاى انديس كواتس و زمان نخهدارى انجام كرفت. جهرت

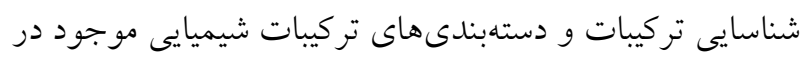

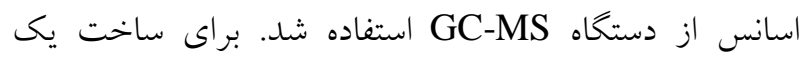

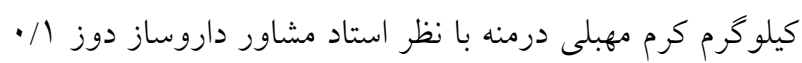

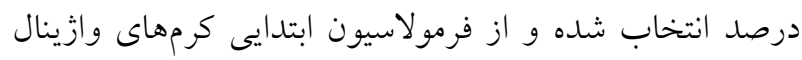

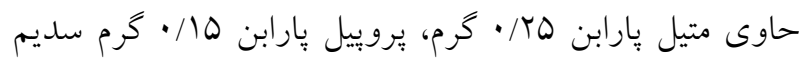

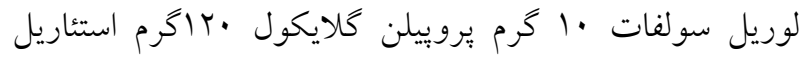

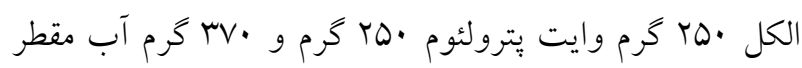

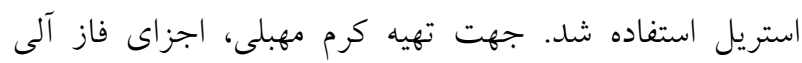

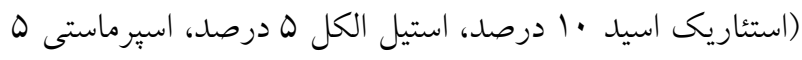

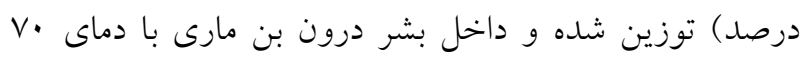

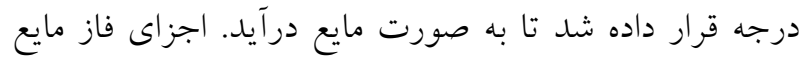

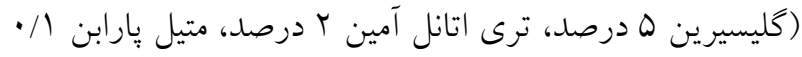

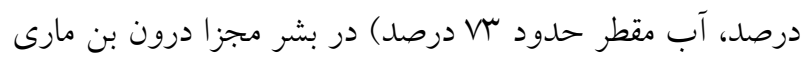

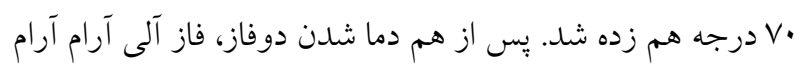

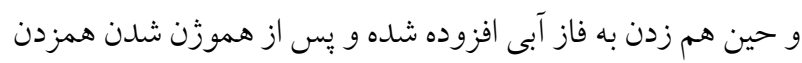

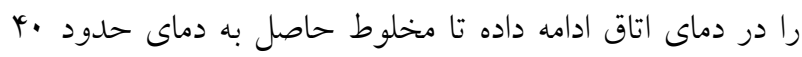

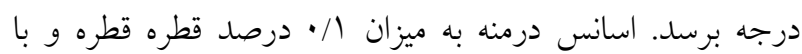

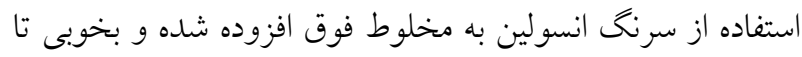

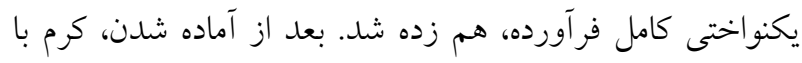

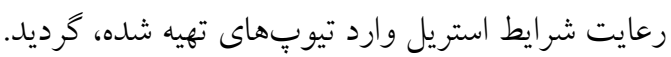

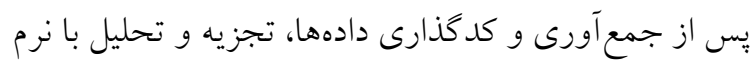

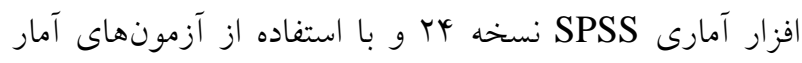

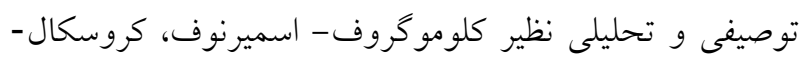

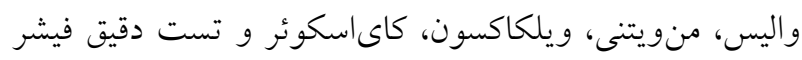

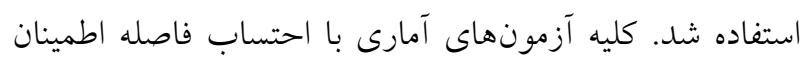

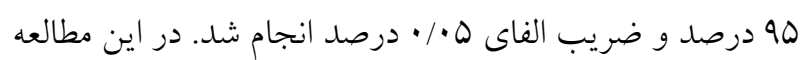

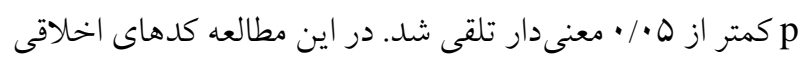

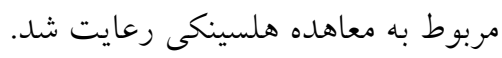

طرز تهيه دارو: جهت جمع آورى گونه Artemisia kulbadica

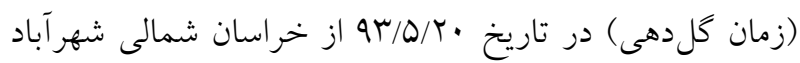

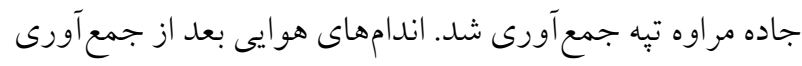

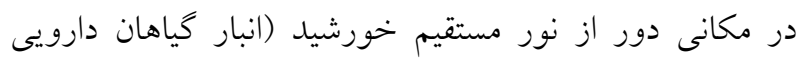
دانشكدهى داروسازى مشهد) و با تهويه مناسب خشكى شد.

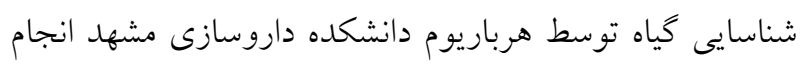

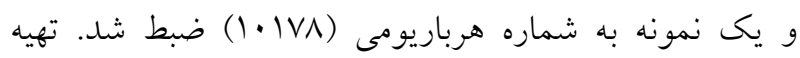

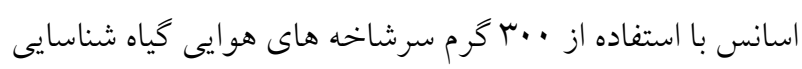

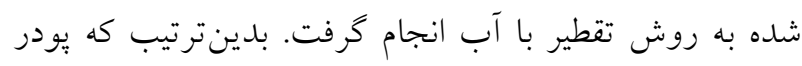

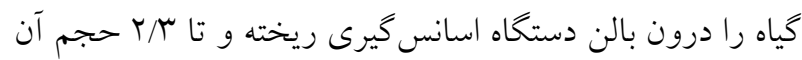

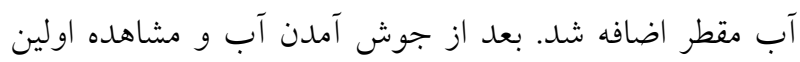

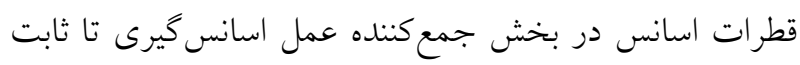
ماندن حجم اسانس در اين بخش، ادامه يِيدا كرد. بعد از بـ بايان

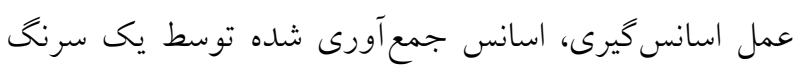

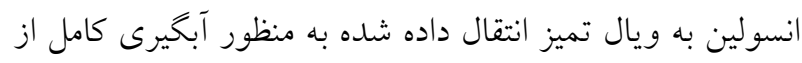

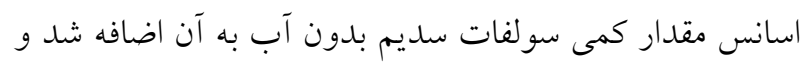

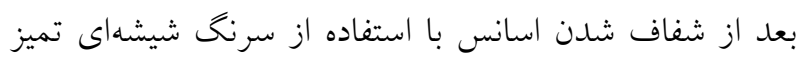

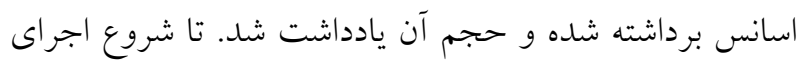

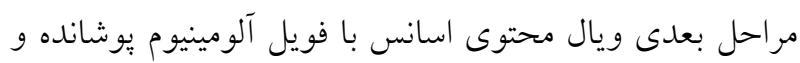

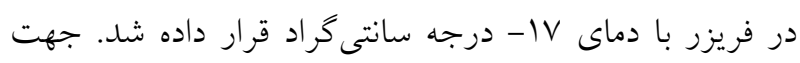

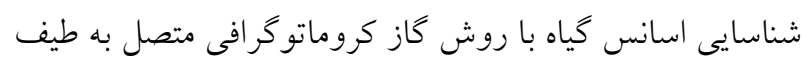

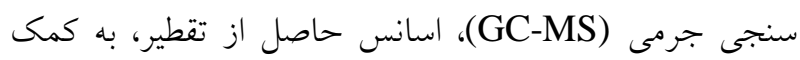

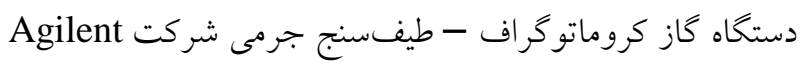

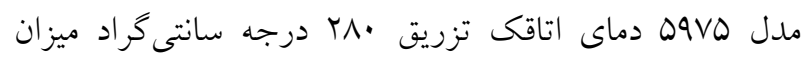

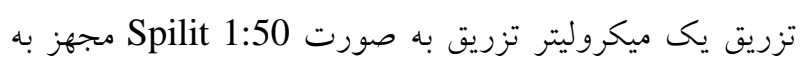

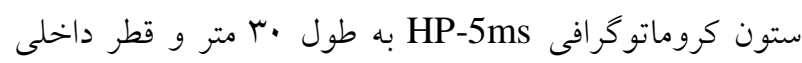

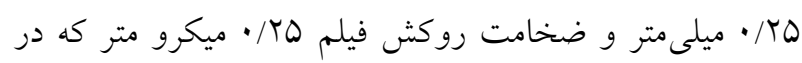

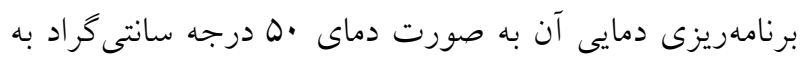

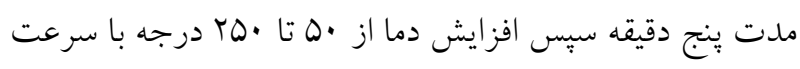

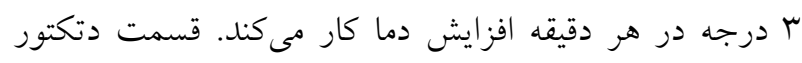

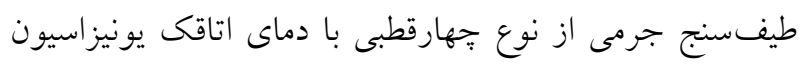

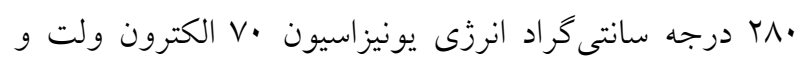

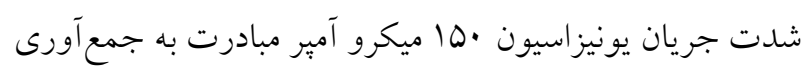


بررسى مقايسهاى تأثير ...

موجود در اسانس از دستكاه GC-MS استفاده شد كه نتايج آناليز

به شرح ذيل مىباشد (جدول شماره انداه د).

جهت شناسايى تركيبات و دستهبندىهاى تركيبات شيميايى

جدول شماره 1- مشخصات و درصد اجزاى موجود در اسانس حاصل از اندام هوايى گونهى Artemisia kulbadica

\begin{tabular}{|c|c|c|c|}
\hline شماره & تركيب & (RI) شاخص نغهدارى (R) & درصد \\
\hline 1 & Santolina triene & $9 \cdot 4$ & $\cdot / 1$ \\
\hline r & Tricyclene & 919 & $\cdot \pi$ \\
\hline r & $\alpha$-Thujene & 948 & $\cdot / r$ \\
\hline r & $\alpha$-Pinene & arr & $\cdot / r$ \\
\hline 0 & Camphene & $94 \wedge$ & $r / \cdot$ \\
\hline 4 & Verbenene & 900 & $\mathrm{~T}$ \\
\hline$v$ & Sabinene & $9 v r$ & $\cdot / 1$ \\
\hline$\wedge$ & $\beta$-Pinene & QVQ & $\cdot / \mu$ \\
\hline 9 & Unknown & qAr & $\cdot / r$ \\
\hline 1. & Yomogi alcohol & $1 \ldots$ & $1 / 1$ \\
\hline 11 & $\alpha$-Terpinene & 1.10 & $\cdot \pi$ \\
\hline ir & 1,8-cineol & 1.48 & $r \Delta / \cdot$ \\
\hline r & Santolina alcohol & 1.49 & $I V / T$ \\
\hline it & $\gamma$-Terpinene & 1.91 & $\cdot / 0$ \\
\hline 10 & cis-Sabinene hydrate & 1.99 &.$/ 1$ \\
\hline 19 & Artemisia alcohol & $1 \cdot 10$ & $\cdot \pi$ \\
\hline iv & Terpinolene & $1 \cdot 1 \mathrm{~V}$ & $\cdot / 0$ \\
\hline 11 & trans-Sabinene hydrate & 1.99 & $\cdot \pi$ \\
\hline 19 & Unknown & llKr & $r / r$ \\
\hline r. & Mentha-2,8-diene-7-ol & $11 T r$ & $\cdot / 1$ \\
\hline ri & Thujyl alcohol = iso-3-thujanol & $111 \mathrm{rr}$ & $r / \mu$ \\
\hline rt & Camphor & 110 & $\cdot / r$ \\
\hline$r$ & Pinocarvone & 1194 & $\cdot / r$ \\
\hline ry & Borneol & $11 \mathrm{v}$. & $r M / 4$ \\
\hline ro & Santolinyl acetate & $11 v 0$ & $\cdot / r$ \\
\hline rq & Terpinen-4-ol & 1111 & 4/9 \\
\hline TV & Unknown & $11 / 4$ & $\Delta / 9$ \\
\hline \ی & Unknown & 11149 & $1 / \pi$ \\
\hline rq & $\alpha$-Terpineol & 1194 & $\cdot / r$ \\
\hline$r$. & Myrtenol & 1194 & $\cdot / r$ \\
\hline$\mu_{1}$ & Myrtenal & 1199 & $\cdot / r$ \\
\hline rr & Verbenone & $r \cdot v$ & $\cdot / 1$ \\
\hline r & Cumin aldehyde & $|r k|$ & $\mathrm{t}$ \\
\hline
\end{tabular}




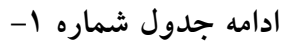

\begin{tabular}{|c|c|c|c|}
\hline شماره & تر كيب & شاخص نخهدارى (RI) & درصد \\
\hline ry & Carvone & IrkT & $\cdot / 1$ \\
\hline ro & Carvotanacetone & iro. & $\cdot / 1$ \\
\hline re & Piperitone & ITOY & $\cdot / \mu$ \\
\hline rv & Carvenone & ITQV & $\mathrm{t}$ \\
\hline ґ & Unknown & ITVG & $1 / \mu$ \\
\hline rq & Isobornyl acetate & $I T \wedge D$ & $\cdot / 0$ \\
\hline r. & Lavandulyl acetate & krq. & $\cdot / \Lambda$ \\
\hline+1 & Unknown & $1 \mathrm{kq \Lambda}$ & $\cdot / \mathrm{V}$ \\
\hline er & Carvacrol & $\mu \cdot v$ & $\cdot / r$ \\
\hline r & Unknown & $1 \pi 11$ & $\cdot / 4$ \\
\hline ky & $\alpha$-Terpenyl acetate & Irka & $\cdot / r$ \\
\hline rQ & Eugenol & Irov & $\cdot 10$ \\
\hline k9 & $\alpha$-Copaene & irVV & $\cdot / 1$ \\
\hline \&V & Methyl cinnamate & $|r \lambda|$ & $\cdot / 1$ \\
\hline ץ & Jasmone<z-> & 1799 & $\cdot / 1$ \\
\hline 49 & $\beta$-Chamigrene & $14 \mathrm{FVO}$ & $\cdot / \mu$ \\
\hline Q. & Germacrene-D & IFAT & $\cdot, 1$ \\
\hline 01 & $\beta$-Selinene & IYAV & $\cdot / 1$ \\
\hline$\Delta r$ & Bicyclogermacrene & lkat & $\cdot / 1$ \\
\hline or & Spathulenol & $10 V 9$ & $\cdot / 9$ \\
\hline$\Delta F$ & $\beta$-Cedrene epoxide & 19K4 & $\cdot / 1$ \\
\hline \multirow[t]{6}{*}{$\Delta \Delta$} & $\begin{array}{c}\gamma \text {-Eudesmol } \\
\text { Grouped compound }\end{array}$ & $|9 \pi|$ & $\cdot / r$ \\
\hline & Monoterpene hydrocarbons & & $\Delta / r$ \\
\hline & Oxygenated monoterpenes & & $v 9 / r$ \\
\hline & Sesquiterpene hydrocarbons & & $\cdot / V$ \\
\hline & Oxygenated sesquiterpenes & & $1 / 1$ \\
\hline & Total identified & & $\wedge \mathrm{V} / \cdot$ \\
\hline
\end{tabular}

RI: The Kovats retention indices relative to C8-C20 n-alkanes were determined on HP-5 ms capillary column. $t$ : trace $<0.05 \%$

كروه همخن بودند (Q • • P>). هم:حنين تفاوت آمارى معنى دارى

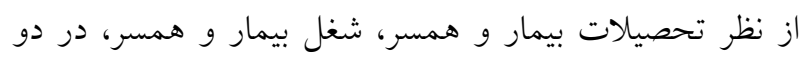

$$
\text { كروه درمانى مشاهده نشد (جدول شماره Y). }
$$

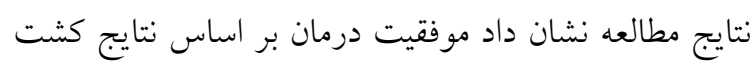

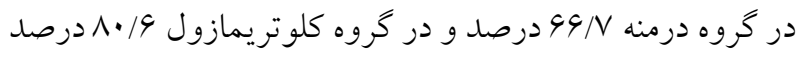

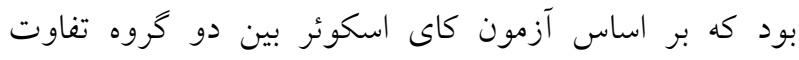

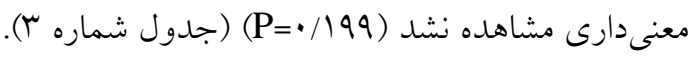

بررسى مشخصات فردى واحدهاى يزوهش نشان داد، ميانخين سنى در گروه درمنه

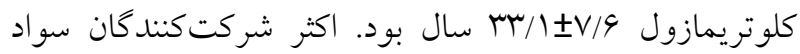

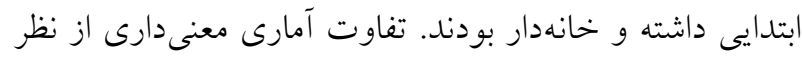

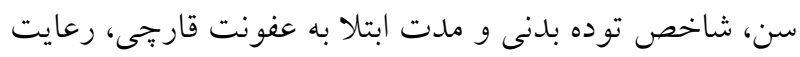

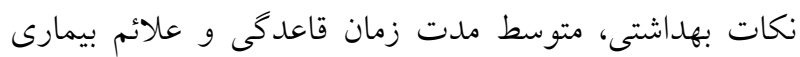

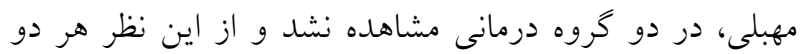




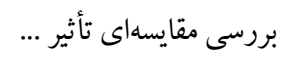

اساس آزمون من ويتنى تفاوت معنى دار نبود (P=•/1/9). آزمون همجنين قبل از درمان ميانخين نمره نشانههاى عفونت

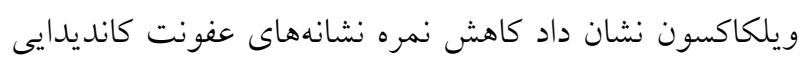

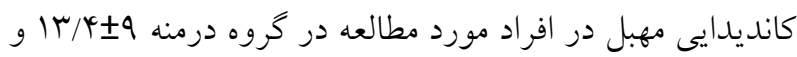

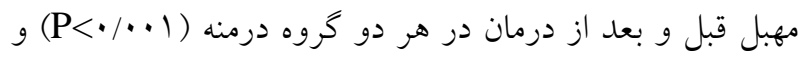

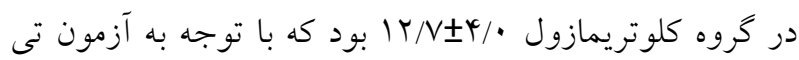

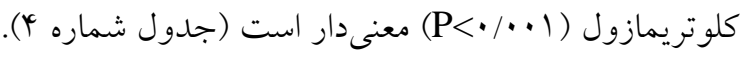

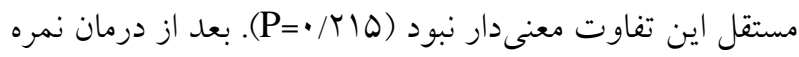

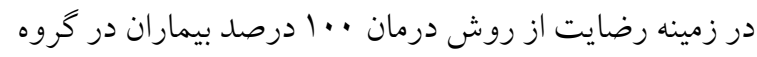

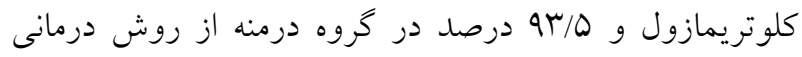
نشانههاى كانديدايى مهبل در افراد مورد مطالعه در خروه درمنه

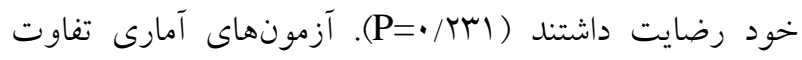

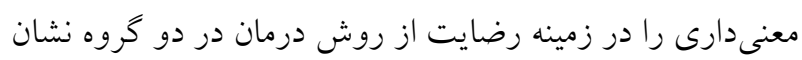

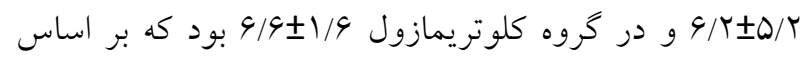

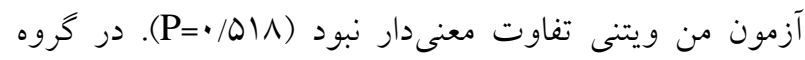

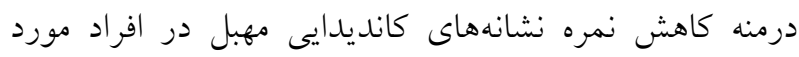

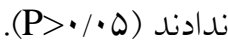
مطالعه /

جدول شماره Y - مشخصات فردى و خانوادگى واحدهاى يزوهش به تفكيك دو گروه

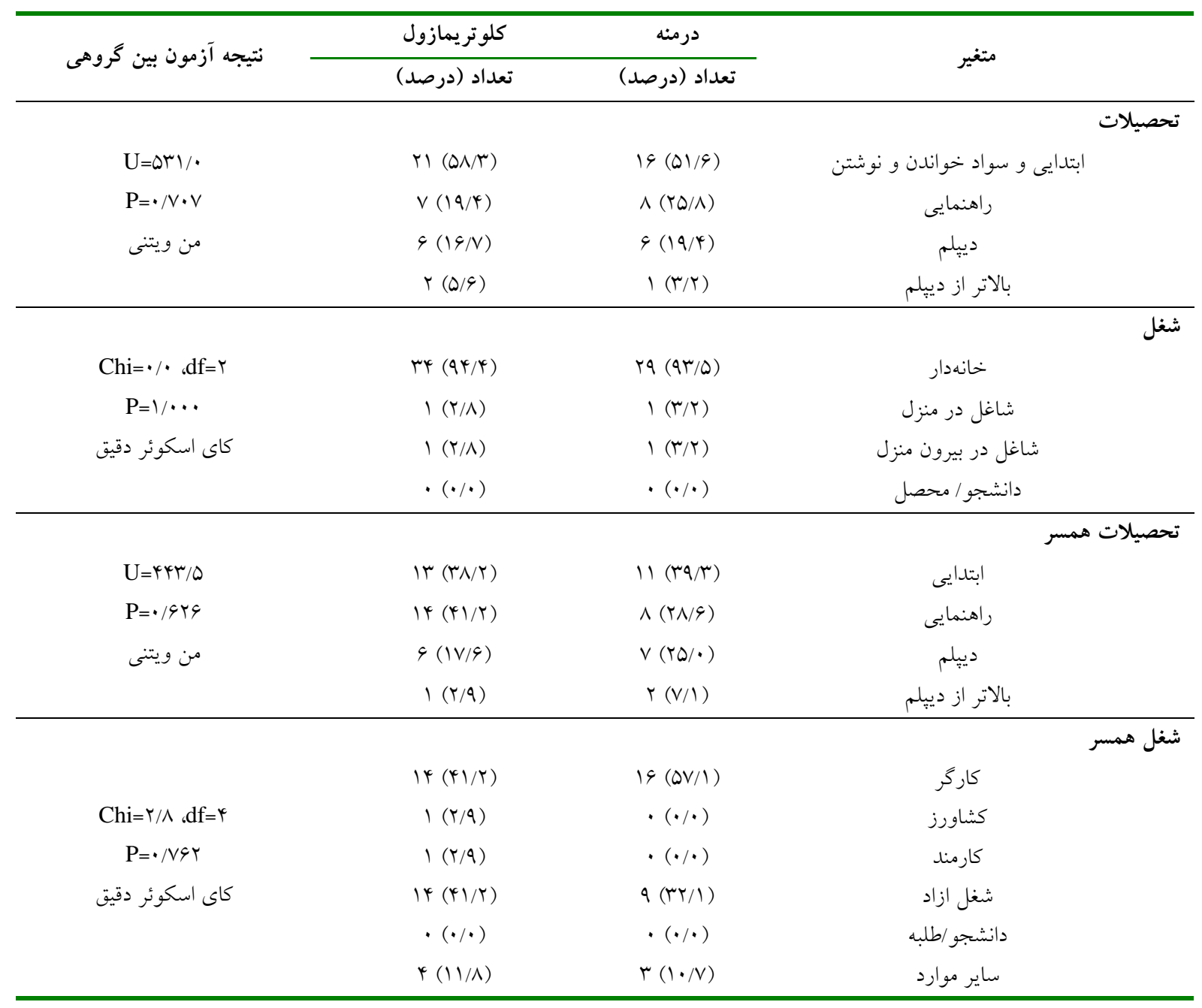


جدول شماره ب- تعداد و درصد زنان مورد مطالعه بر حسب موفقيت درمان به تفكيك داروى مورد استفاده بعد از درمان

\begin{tabular}{|c|c|c|c|}
\hline نتيجه آزمون بين گروهى & كلوتر يمازول & 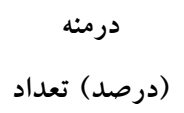 & متغير \\
\hline$\chi=1 / \vee d \mathrm{df}=1$ & & & نتيجه كشت ترشحات \\
\hline $\mathrm{P}=\cdot / 199$ & $V(19 / 4)$ & $1 \cdot(\Gamma / \Gamma)$ & مثبت \\
\hline كاى اسكوئر & rq $(1 \cdot / 9)$ & $r \cdot(94 / \mathrm{V})$ & 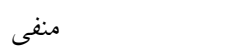 \\
\hline$\chi=I / v d \mathrm{df}=1$ & & & نتيجه كشت KOH \\
\hline $\mathrm{P}=\cdot / 199$ & $V(19 / 4)$ & $1 \cdot(\mu r / \mu)$ & مثبت \\
\hline كاى اسكوئر & rq $(\Lambda \cdot / 9)$ & $r \cdot(99 / \mathrm{V})$ & منفى \\
\hline
\end{tabular}

جدول شماره F- ميانخين و انحر اف معيار نمره نشانههاى كانديداى مهبلى واحدهاى بروهش قبل و بعد از درمان به تفكيك داروى مورد استفاده

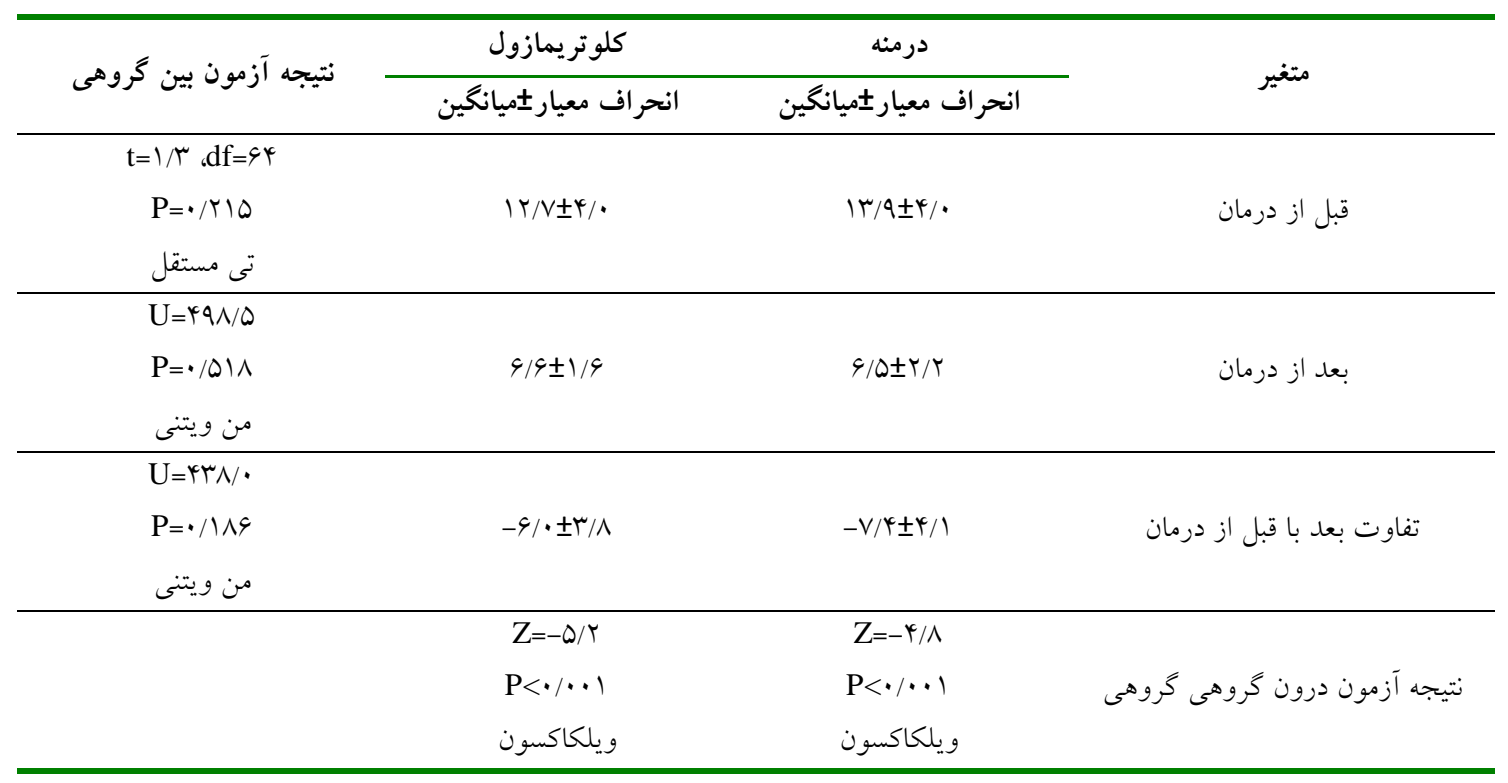

مكرر وجود دارد، لذا در بسيارى از تحقيقات جديد به بررسى

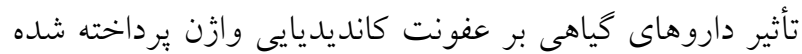
است [TN، T [YV]. بر اين اساس مطالعه حاضر نيز به منظور مقايسه تأثير كرم مهبلى درمنه و كلوتريمازول بر عفونت كانديدايى مهبل بر روى زنان غيرباردار واقع در سنين بارورى شهر مشهد در سال

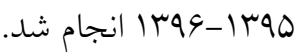
با توجه به نتايج به دست آمده از اين مطالعه موفقيت درمان بر اساس نتايج آزمايشخاهى در هر دو كروه درمنه و كلوتريمازول معنى دار بود. همجنين نتايج، تفاوت نتايج كشت و

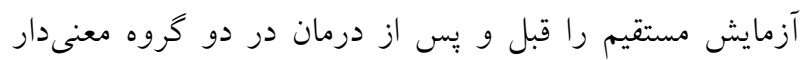

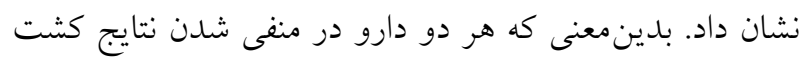

كانديديازيس ولو و وازن يكى از شايعترين علل مراجعه زنان به كلينيكهاى زنان - مامايى مىباشد و ه درصد اين زنان موارد عود مكرر اين عفونت را تجربه مى كنند. در اين بيماران

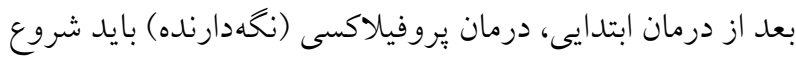

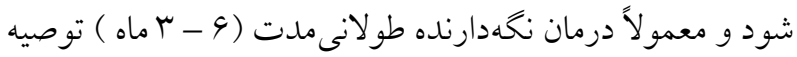
مىشود. با توجه به مصرف طولانىمدت درمان نخهدارنده، امروزه تمايل به سمت استفاده از داروهاى خوراكى بوجود آمده بهوديه است ولى جون اين درمانها، توكسيسيته سيستميك، بخصوص در موارد مصرف طولانى مدت را به فرد تحميل مى كنند و نيز در مواردى عدم ياسخ / مقاومت دارويى بعد از عودها و درمانهاى 
بررسى مقايساى تأثير ...

كلوتريمازول مشابه با فلوكونازول بوده است. بدينمعنى كه

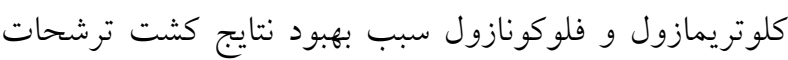

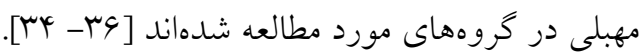
از جمله مطالعه غير همسو با بزّوهش حاضر مطالعه روزبهانى و مورد

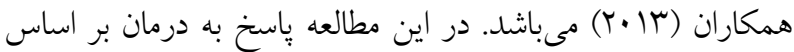
نتايج كشت ترشحات مهبلى در كروه مصرف كننده كيسول خوراكى

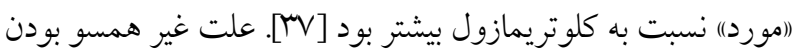
يزوهش روزبهانى با مطالعه حاضر مى تواند ناشى از دوز تجويزى و

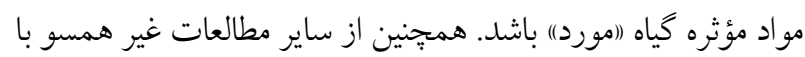

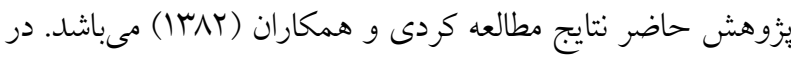
اين مطالعه اختلاف معنىدارى در پياسخ به درمان بر اساس كثت

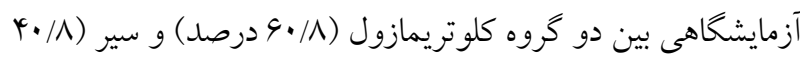

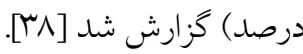

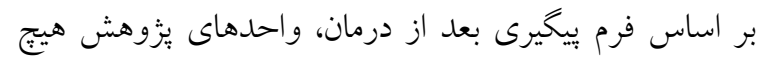

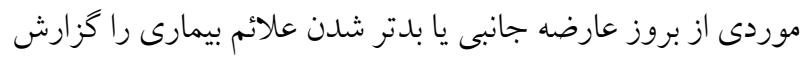

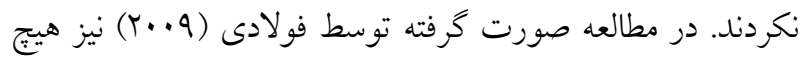
موردى از بروز عارضه جانبى در زمنيه مصرف كرم مهبلى آويشن

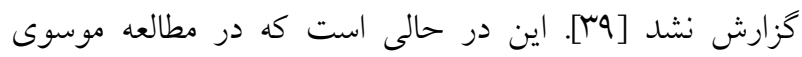

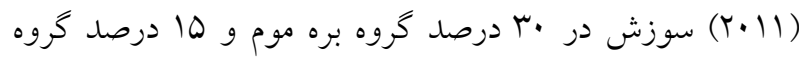

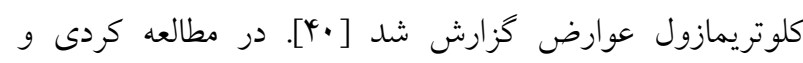

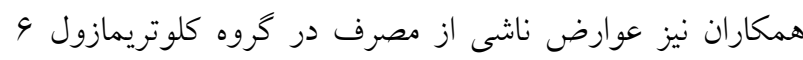

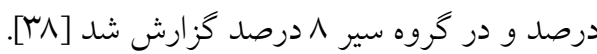
از نقاط قوت مطالعه مىتوان به سه سوكور بودن مطالعه، استفاده

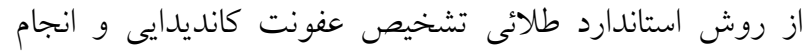

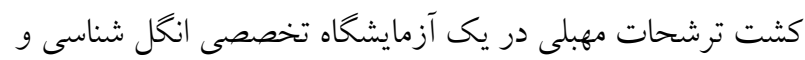

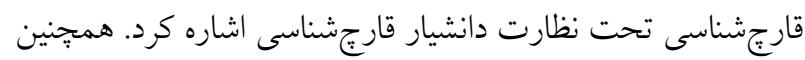

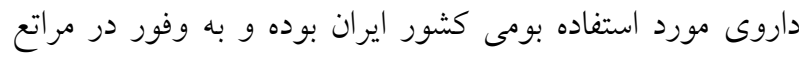

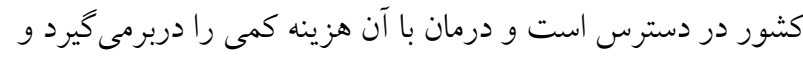

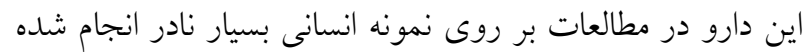

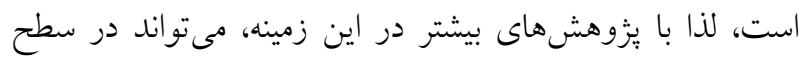

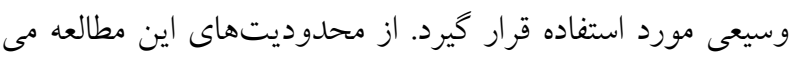

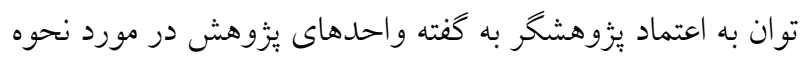

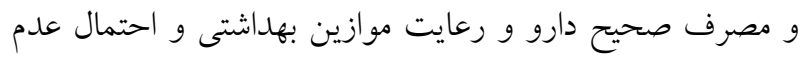

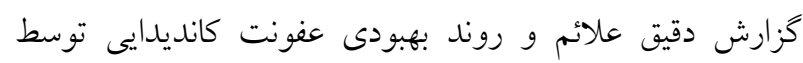

ترشحات مهبلى و آزمايش مستقيم مؤثر بودند. در اين راستا مطالعه فولادى و همكاران با نتايج مطالعه حاضر همسو مى بـاشد.

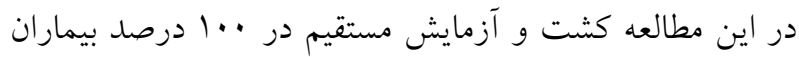

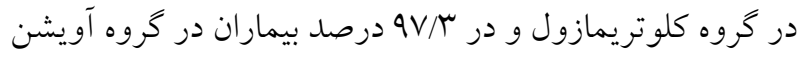

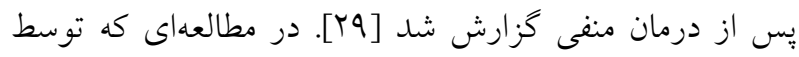

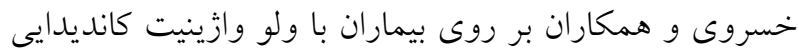

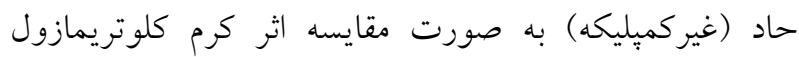

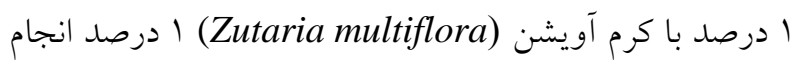

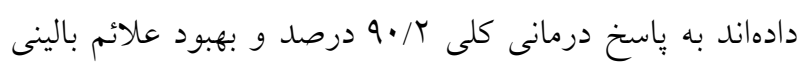

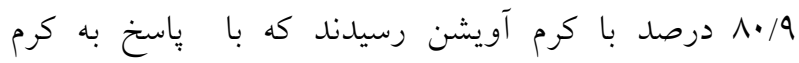
كلوتريمازول مشابه بوده است [•rّ]. متأسفانه اين تنها مطالعه

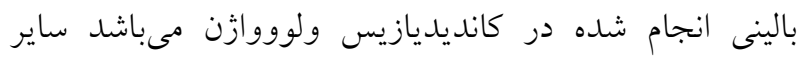

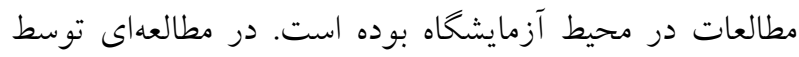

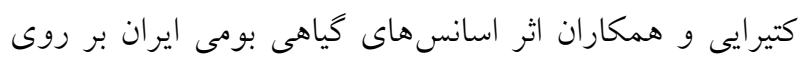

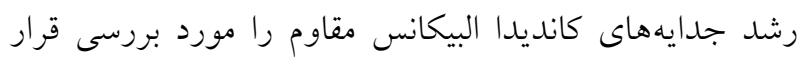

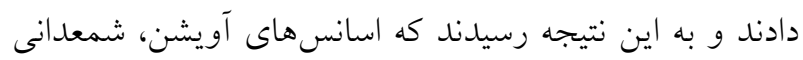

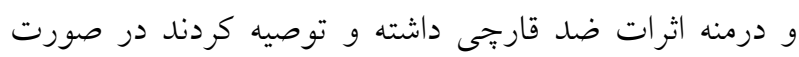
وجود مقاومت جدايههاى مختلف كانديدا البيكانس به داروهاى

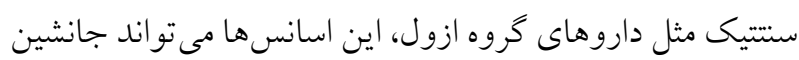

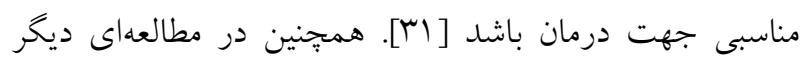

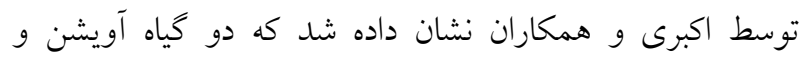

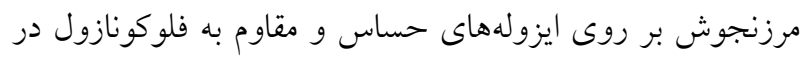

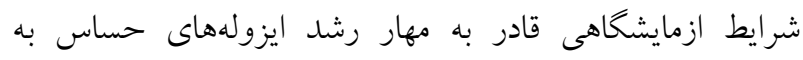

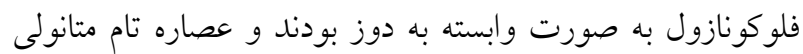

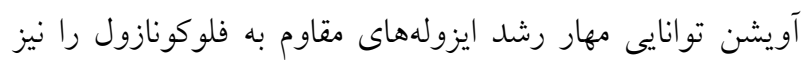

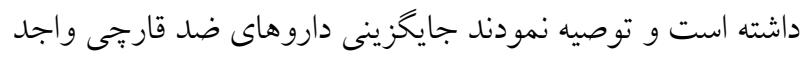
آثار جانبى شديد با تركيبات دارويى طبيعى منطقى به نظر مى نـرسد

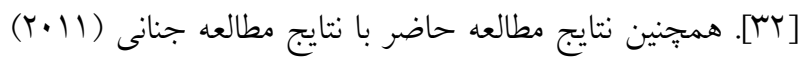

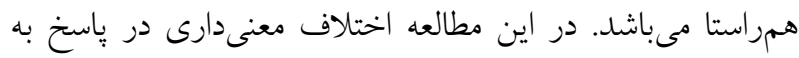

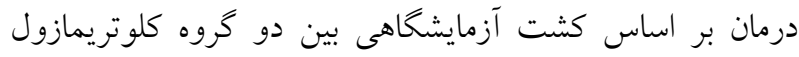

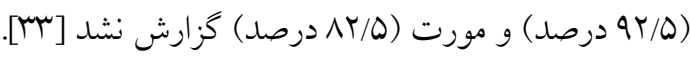
همجِنين نتايج مطالعه حاضر با مطالعه سوبل (Y) (sobel)

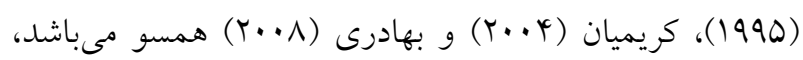

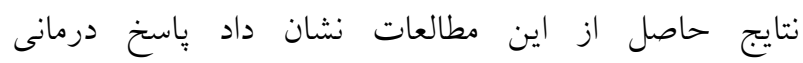


شدند، و اثرات درمانى اين دو دارو در مقايسه با هم مشابه بود.

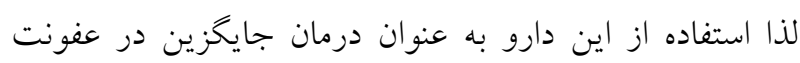

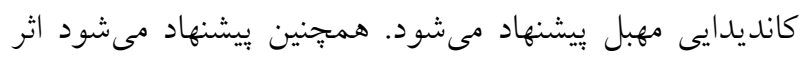
گياه درمنه در اشكال و دوزهاى مختلف در درمان عفونت كانديديايى مهبل و همجنين در درمان عفونت راجعه كانديديايى

$$
\text { تشرد بررسى قرار كيرد. }
$$

اين مقاله بخشى از ياياننامه كارشناسى ارشد مامايى و

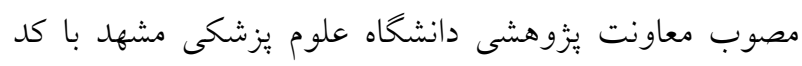
اخلاق IRCT2016121831454N1 مراتب تشكر و قدردانى خود را از معاونت يزٔوهشى دانشگاه علوم يزشكى مشهد به دليل تأمين بودجه اين مطالعه و مراكز

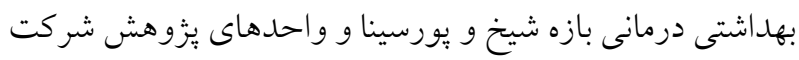

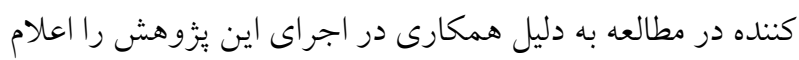

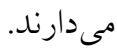

1. R. N. Comparison the effectiveness of cinnamon oral capsule with clotrimazole vaginal cream for treatment of women with vaginal candidiasis at centers of Shahid Beheshti University Medical Sciences 2013. Masters Thesis: Tehran, Iran: Shahid Beheshti University Faculty of Midwifery and Nursing. 2013.

2. Fouladi Z, Afshari P, Gharibi T and Dabbagh M. The comparison of Zataria multiflora boiss (Avishan Shirazi) and Clotrimazol vaginal cream in the treatment of candidiasis vaginitis. ISMJ. 2009; 12 (12): 214-24.

3. Mousavi M, Keshavarz T, Montaseri H, Pakshir $\mathrm{K}$, Yazdani $\mathrm{M}$ and Zare N. A comparative study on
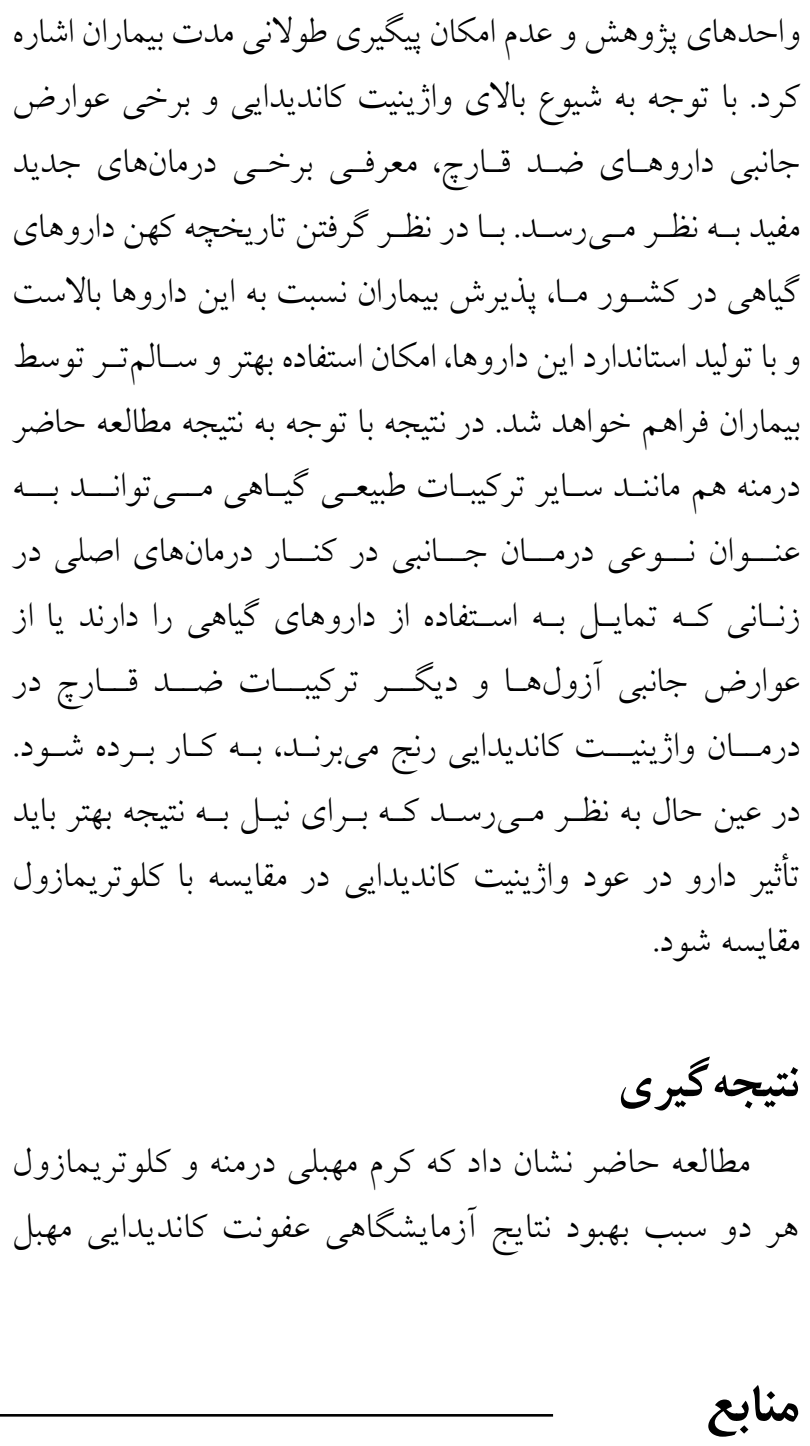

the therapeutic effect of the propolis vaginal cream and clotrimazole on candida vulvovaginitis in reproductive aged women. J. Isfahan Med. Sch. 2011; 28 (117): 1-9.

4. Banaean-Boroujeni S, Rasti-Boroujeni M, Moghim H, Validi M, Mobini G and Kazemian A. In vitro effect of honey on Candida albicans and lactobacillus. J. Shahrekord Univ. Med. Sci. 2010; 11 (4): 52-8.

5. Seyed Mazhari $M$ and Tudeh Fallah F. A comparative study of vaginal candidiasis in pregnant and non-pregnant women. J. the Nursing Academy of the Islamic Republic of Iran. 2009; 9 (1): 18-20. 
بررسى مقايسهاى تأثير ...

6. Gharekhani P, Sadatin A. Cardinal manifestations \& management of diseases (CMMD). Tehran: Noure Danesh. 2005.

7. Dunnihoo D. Fundamentals of gynecology and obstetrics. New York: Lippincott. 2010.

8. Wilson E. Recurrence of Vulvovaginal candidacies during pregnancy, obstet, Gynecol. 2002; 48 (4): 491-494.

9. Break J. Break and Novak's gynecology. Volum

1 Chapter 14. Genetic Urinary infection and Sexuality Transmitted Diseases, Lippincott Williams \& Wilkins. 14th ed. 2012, pp: 545-7.

10. Saebi S. Clinical Iran Generic Drugs. 5th ed, Aeig. 2001, p: 243.

11. Jafari Nodoushan A, Dehghani $M$ and Mirbagheri S. In vitro Antifungal Effect of Aqueous Garlic (Allium Sativum) Extract and its Combination with fluconazole against five common clinical candida isolated from candidiasis lesions. $J$. Kerman Univ. Med. Sci. 2007; 14 (3): 153-62.

12. Katiraee F, Eidi S, Bahonar A, Zarrinfar $\mathrm{H}$ and Khosravi A. Comparision of MICs of some Iranian Herbal Essences Against Azole Resistance and Azole Susceptible of Candida albicans. J. Med. Plants 2008; 7 (27): 37-44.

13. Horiuchi $K$, Shiota $S$, Kuroda $T$, Hatano $T$, Yoshida T and Tsuchiya T. Antimicrobial activity of Oleanolic Acid from Saliva officinalis and related compounds on vancomycin-resistant enterococci. Biol. Pharm. Bull. 2007; 30 (6): 1147-9.

14. Mousavi Z, Keshavarz T, Pakshir K, Yazdani M, Zare N and Afrouzan H. A Comparative Study on the Therapeutic Effect of the Propolis Vaginal Cream and Clotrimazole on Candida Vulvovaginitis in Reproductive Aged Women. J. Isfahan Medical School 2011; 28 (117): 1-9.

15. Santomauro F, Donato R, Sacco C, Pini G, Flamini $G$ and Bilia A. Vapour and Liquid-Phase Artemisia annua Essential Oil Activities against Several Clinical Strains of Candida. Planta Med. 2016; 82 (11-12): 1016-20.
16. Juteau F, Jerkovic I, Masotti V, Milos M, Mastelic $\mathrm{J}$ and Bessiere $\mathrm{J}$. Composition and antimicrobial activity of the essential oil of Artemisia absinthium from Croatia and France. Planta Med. 2003; 69 (2): 158-61.

17. Masoudi S, Rustaiyan A and Vahedi M. Volatile oil constituents of different parts of Artemisia chamaemelifolia and the composition and antibacterial activity of the aerial parts of Aturcomanica from Iran. Nat. Prod. Commun. 2012; 7 (11): 1519-22.

18. Choi E, Park H, Lee J and Kim G. Anticancer, antiobesity, and anti-inflammatory activity of Artemisia species in vitro. J. Tradit. Chin. Med. 2013; 33 (1): 92-7.

19. Habibi Z, Ghanian S, Ghasemi S and Yousefi M. Chemical composition and antibacterial activity of the volatile oil from seeds of Artemisia annua L. from Iran. Nat. Prod. Res. 2013; 27 (2): 198-200.

20. Kazemi M, Dakhili M, Dadkhah A, Yasrebifar $\mathrm{Z}$ and Larijani K. Composition, antimicrobial and antioxidant activities of the essential oil of Artemisia kermanensis Podl, an endemic species from Iran. J. Med. Plants Res. 2011; 5 (18): 4481-6. 21. Ghlissi Z, Sayari N, Kallel R, Bougatef A and Sahnoun Z. Antioxidant, antibacterial, antiinflammatory and wound healing effects of Artemisia campestris aqueous extract in rat. Biomed. Pharmacother. 2016; 84: 115-22.

22. Hakimi Meybodi MH, Afkhami Aghad M and Mir Jalili F. An investigation into biological activities of A. persica's essential oil. Pajouhesh \& Sazandegi 2004; 16 (4): 2-5.

23. Naeini A, Naseri $M$, Kamalinejad $M$, Khoshzaban F, Rajabian T, Nami H and et al . Study on Anti_ Candida Effects of Essential Oil and Extracts of Iranian Medicinal Plants, In vitro. $J$. Med. Plants 2011; 2 (38): 163-172.

24. Obistioiu D, Cristina R, Schmerold I, Chizzola R, Stolze $\mathrm{K}$ and Nichita I. Chemical characterization by GC-MS and in vitro activity against Candida albicans of volatile fractions

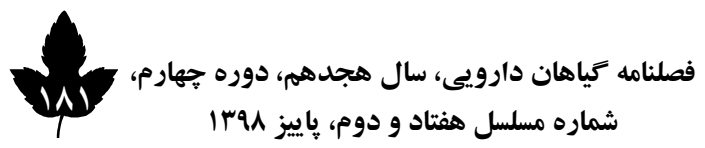


prepared from Artemisia dracunculus, Artemisia abrotanum, Artemisia absinthium and Artemisia vulgaris. Chem. Cent. J. 2014; 8 (1): 6.

25. Teamori $F$ and Rezae $S$. Iranian generic drugs with nursing and care measures. Tehran: Arian teb. 2015, p: 1262.

26. Tan J, Zhang J, Chen W, Sun Y, Wan Z and Li R. The A395T mutation in ERG11 gene confers fluconazole resistance in Candida tropicalis causing candidemia. Mycopathologia 2015; 179 (3-4): 213-8.

27. White D, Johnson E and Warnock D. Management of Persistent Vulvovaginal Candidiasis due to azole resistant Candidia glabralata. Genitourin. Med. 1993; 6 (2): 112-4.

28. Mikamo H, Kawazoe K, Sato Y, Hayasaki Y and Tamaya T. Comparative study on the effectiveness of antifungal agents in different regimens against vaginal Candidiasis. Chemotherapy 1998; 44 (5): 364-81.

29. Fouladi Z, Afshari P, Gharibi $T$ and Dabbagh M. The comparison oF Zataria multiflora boiss (Avishan Shirazi) and Clotrimazol vaginal cream in the treatment of candidiasis vaginitis. ISMJ. 2009; 12 (3): 214-24.

30. Khosravi AR, Eslami AR, Shokri H, Kashanian M. Zataria multiflora cream for the treatment of acute vaginal candidiasis. International Journal of Gynecology and Obstetrics 2008; 101 (2): 201-202. 31. Katiraee F, Eidi S, Bahonar A, Zarrinfar H, Khosravi A. Comparision of MICs of some Iranian Herbal Essences Against Azole Resistance and Azole Susceptible of Candida Albicans. J. Med Plants 2008; 3 (27): 37-44.

32. Akbari S. Antifungal Effects of Thyme and Marjoram Plant Extract on Clinical Isolates of Candida Albic Acid Resistant and Fluconazole Sensitive. J. Med. Plants (Special Issue of Plants with Antimicrobial Effects). 2006; 1 (Sup. 3): 53-62.

33. Janani F, Delfan B, Toulabi T, Ebrahim zadeh F and Motamedi M. Comparative study of effect of myrtus vaginal cream and clotrimazol vaginal cream in the treatment of vaginal candidiasis. Scientific Magazine Yafte. 2011; 13 (1): 32-41.

34. Sobel J, Brooker D, Stein G, Thomason J, Wermeling D and Bradley B. Single oral dose fluconazole compared with conventional clotrimazole topical therapy of Candida vaginitis. American J. Obstetrics and Gynecol. 1995; 172 (4): 1263-8.

35. Kariman N, Afrakhteh M, Valaii N and Ahmadi M. Comparison of the effect of fluconazole and clotrimazole in the treatment of vulvovaginal candida albicans. J. Kermanshah University of Medical Sciences 2008; 6 (3): 9-16.

36. Bahadori F, Diba K, Yekta $Z$ and Namaki A. Comparison of Fluconazole and Clotrimazole in the Treatment of Acute Candida Albicans Vulvovaginitis. J. Family and Reproductive Health 2008; 2 (4): 179-83.

37. Roozbahani F, Kariman N, Mojab F and Nasiri M. Effect of Myrtus communis capsule on vaginal candidiasis treatment. Pajoohandeh J. 2013; 18 (5): 242-9.

38. Kordi M, Rakhshandeh $\mathrm{H}$ and Gholami $\mathrm{H}$. Comparison of the effect of garlic extract vaginal douche and clotrimazol vaginal cream in the treatment of women with vaginal candidiasis. Iranian Journal of Obstetrics, Gynecology and Infertility 2005; 8 (2): 33-42.

39. Fouladi Z, Afshari P, Gharibi $T$ and Dabbagh M. The comparison oF Zataria multiflora boiss (Avishan Shirazi) and Clotrimazol vaginal cream in the treatment of candidiasis vaginitis. Iranian South Medical J. 2009; 12 (3): 214-24.

40. Mousavi M, Pakshir K, Yazdani M, Zare N and Afrouzan $\mathrm{H}$. Comparison of the effects of bee propolis cream clotrimazole vaginal cream in the treatment of fungal infections of the vagina in women of childbearing age. J. Isfahan Medical School 2011; 28 (117): 1099-1107. 
بررسى مقايسهاى تأثير ....

\title{
A Comparative Study on the Effect of Artemisia and Clotrimazole Vaginal Cream on Vaginal Candida Infection on Non-Pregnant Women in Fertile Age in Mashhad in 2016-2017: A Triple Blind Clinical Trial
}

\author{
Ebrahimzadeh Zgemi S (Ph.D.) ${ }^{1}$, Golmakani N (M.Sc.) ${ }^{2}$, Asili MJ (Ph.D.) ${ }^{3}$, Naseri A (Ph.D.) ${ }^{4}$, \\ Mohebbi Dehnavi Z (M.Sc.) ${ }^{5}$, Kamali Z (M.Sc.) ${ }^{6}$, Saber mohammad A (M.Sc.) ${ }^{7^{*}}$
}
1- PhD student in Reproductive Health, Faculty member of midwifery, Department of Midwifery, Mashhad University of Medical Sciences, Mashhad, Iran
2- Assistant professor of Midwifery, Faculty Member of Mashhad University of Medical Sciences, Department of midwifery, Mashhad, Iran
3- Assistant professor of Pharmacy, Faculty member of Mashhad University of Medical Sciences, Department of Pharmacy, Mashhad, Iran
4- Assistant professor of Parasitology \& Mycology, Faculty member of Mashhad University of Medical Sciences, Department of Parasitology \& Mycology, Mashhad, Iran
5- Master of Midwifery, Faculty of Nursing and Midwifery, Isfahan University of Medical Sciences, Isfahan, Iran
6- Master of Midwifery, Department of Midwifery, School of Nursing and Midwifery, Mashhad University of Medical Sciences, Mashhad, Iran
7- Master of Midwifery, Department of Midwifery, Student Research Committee, Mashhad University of Medical Sciences, Mashhad, Iran
*Corresponding author: Department of Midwifery, Student Research Committee, Mashhad
University of Medical Sciences, Mashhad, Iran
Tel: +98-915-9516438
Email: saberma921@mums.ac.ir

\begin{abstract}
Background: About $75 \%$ of women experience at least one vulvovaginal candidiasis during their life. Clotrimazole is an antifungal drug that has side effects along with its therapeutic properties. For this reason, a large percentage of women tend to use natural herbal treatments. Artemisia is an anti-fungal activity.

Objective: The aim of this study was to compare the effect of Artemisia vulgaris and Clotrimazole on vaginal candida infection on non pregnant women in fertility ages.

Methods: This study is a triple blind clinical trial performed on 72 non-pregnant women in Mashhad in 2016-2017 years. The research units were divided into two groups (receiving Artemisia Vaginal Cream and receiving Clotrimazole Vaginal Cream). After confirmation of candidacy and having consent for participation, by random allocation and treated for seven nights. After the treatment, the data was analyzed using SPSS software (version 24).

Results: After treatment, the score for candidate vaginal symptoms was not significant in Artemisia and Clotrimazole group based on Mann-Whitney test $(P=\mathbf{0 . 5 1 8})$. The Wilcoxon test showed a significant reduction in the symptoms of vaginal candida infection before and after treatment in both groups of Artemisia $(\mathrm{P}<0.001)$ and clotrimazole $(\mathrm{P}<0.001)$.

Conclusion: Due to the similar effects of Artemisia and Cholitrimazole, Artemisia can be used as an alternative treatment for vaginal candidiasis due to less side effects.
\end{abstract}

Keywords: Artemisia, Candidiasis, Clotrimazole, Fertility age, Infection, Women

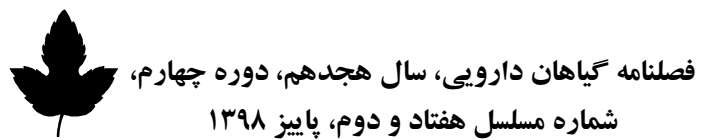

